PHYSICAL REVIEW D 93, 044047 (2016)

\title{
Spinning black holes in Einstein-Gauss-Bonnet-dilaton theory: Nonperturbative solutions
}

\author{
Burkhard Kleihaus, ${ }^{1}$ Jutta Kunz, ${ }^{1}$ Sindy Mojica, ${ }^{1}$ and Eugen Radu ${ }^{2}$ \\ ${ }^{1}$ Institut für Physik, Universität Oldenburg, Postfach 2503, D-26111 Oldenburg, Germany \\ ${ }^{2}$ Departamento de Fisica da Universidade de Aveiro and I3N, \\ Campus de Santiago, 3810-183 Aveiro, Portugal \\ (Received 20 November 2015; published 17 February 2016)
}

\begin{abstract}
We present an investigation of spinning black holes in Einstein-Gauss-Bonnet-dilaton (EGBd) theory. The solutions are found within a nonperturbative approach, by directly solving the field equations. These stationary axially symmetric black holes are asymptotically flat. They possess a nontrivial scalar field outside their regular event horizon. We present an overview of the parameter space of the solutions together with a study of their basic properties. We point out that the EGBd black holes can exhibit some physical differences when compared to the Kerr solution. For example, their mass is always bounded from below, while their angular momentum can exceed the Kerr bound. Also, in contrast to the Kerr case, the extremal solutions are singular, with the scalar field diverging on the horizon.
\end{abstract}

DOI: 10.1103/PhysRevD.93.044047

\section{INTRODUCTION}

The Einstein-Gauss-Bonnet-dilaton (EGBd) gravity is one of the most interesting and best motivated extensions of general relativity (GR). This model modifies the EinsteinHilbert action by adding a (real) scalar field nonminimally coupled to the Gauss-Bonnet (GB) invariant. The resulting theory possesses a number of attractive features. First, the EGBd model is the only theory of gravity with quadratic curvature terms in the action leading to second order equations of motion. Second, the terms in the EGBd action naturally occur in the framework of the low-energy effective string theories, see, e.g., [1], in which case the scalar field can be seen as the string dilaton. Moreover, the EGBd gravity can be considered as a particular case of Horndeski gravity [2], a theory which attracted recently a lot of interest. A review of these aspects in a more general context can be found in the recent work [3].

Similar to the GR case, the EGBd model does not possess particlelike soliton solutions [4]. However, there are black hole $(\mathrm{BH})$ solutions, which intrinsically violate the "no hair" conjecture, possessing always a nonvanishing scalar field [7]. The study of BH solutions of the EGBd gravity has started with the work [8,9], where the generalization of the Schwarzschild solutions was found in closed form within a perturbative approach. The fully nonperturbative solution was constructed numerically in [10-14]. Perhaps the most interesting result there is the existence of a minimal mass for the static BHs, which is set by the GB coupling constant.

In analogy to the GR case, these solutions should possess spinning generalizations. However, their study is much more involved, and mainly perturbative results have been reported in the literature. Here the most advanced results obtained so far are those in the recent work [15]. That paper reports an approximate construction of the spinning solutions in EGBd theory which is of fifth order in the black hole spin and of seventh order in the GB coupling constant (for previous work in this direction, see [14,16,17]).

The perturbative approach has the advantage to lead to closed form expressions of the solutions and to allow for a simple and direct computation of various quantities of interest. At the same time, it is clear that a number of important features (in particular those occurring in the fast spinning regime of the solutions) cannot be caught within this framework.

A different approach has been taken in Ref. [18] which has given a first discussion of the spinning solutions in EGBd theory within a nonperturbative framework. There, the field equations were solved numerically, and the basic properties of the solutions were compared with those of their GR counterparts. Perhaps the most exciting result reported in [18] is that the BHs in EGBd theory can slightly exceed the Kerr bound [19]. Moreover, the innermoststable-circular-orbits (ISCOs) can differ from the respective Kerr values. Also, as discussed in [20], the quadrupole moment of EGBd black holes can be considerably larger than in the GR case.

The main purpose of this work is to provide a detailed description of the (nonperturbative) spinning BHs in EGBd theory reported in [18], with special emphasis on the construction of solutions together with their domain of existence. The paper is organized as follows. In Sec. II we describe the EGBd theory, the Ansatz and the boundary conditions taken. We also introduce physical quantities and discuss the numerical procedure together with the known solutions. This general framework is used in Sec. III, where we present a discussion of the solutions. Finally, in Sec. IV we summarize our results and enumerate a number of research directions that can be addressed in the future. In 
Appendix A we provide some details on a set of EGBd critical solutions which form a part of the boundary of the domain of existence, while Appendix B contains a discussion of the issue of extremal solutions within the near horizon formalism.

\section{THE GENERAL FRAMEWORK}

\section{A. The action}

The Einstein-Gauss-Bonnet-dilaton (EGBd) system is described by the following action [21]

$$
S=\frac{1}{16 \pi} \int d^{4} x \sqrt{-g}\left[R-\frac{1}{2}\left(\partial_{\mu} \phi\right)^{2}+\alpha e^{-\gamma \phi} R_{\mathrm{GB}}^{2}\right],
$$

where $\phi$ is a (real) scalar field, and $\gamma$ and $\alpha$ are input parameters of the theory. Also,

$$
R_{\mathrm{GB}}^{2}=R_{\mu \nu \rho \sigma} R^{\mu \nu \rho \sigma}-4 R_{\mu \nu} R^{\mu \nu}+R^{2}
$$

is the GB term [22]. For the chosen conventions, the (positive) GB coupling parameter [23] $\alpha$ is dimensionful and, specifically, it has dimensions of (length) ${ }^{2}$. Moreover, although all solutions reported in this work have $\gamma=1$, we shall keep its value arbitrary in all general relations.

Varying the action (1) with respect to $g_{\mu \nu}$, we obtain the equations for the metric tensor

$$
\begin{aligned}
E_{\mu \nu}= & G_{\mu \nu}-\frac{1}{2} T_{\mu \nu}^{(\phi)} \\
& +\alpha e^{-\gamma \phi}\left[H_{\mu \nu}+4\left(\gamma^{2} \nabla^{\rho} \phi \nabla^{\sigma} \phi-\gamma \nabla^{\rho} \nabla^{\sigma} \phi\right) P_{\mu \rho \nu \sigma}\right] \\
= & 0,
\end{aligned}
$$

where

$$
\begin{aligned}
G_{\mu \nu} & =R_{\mu \nu}-\frac{1}{2} g_{\mu \nu} R, \quad T_{\mu \nu}^{(\phi)}=\nabla_{\mu} \phi \nabla_{\nu} \phi-\frac{1}{2} g_{\mu \nu}(\nabla \phi)^{2}, \quad \text { and } \\
H_{\mu \nu} & =2\left(R R_{\mu \nu}-2 R_{\mu \rho} R_{\nu}^{\rho}-2 R^{\rho \sigma} R_{\mu \rho \nu \sigma}+R_{\mu}^{\rho \sigma \lambda} R_{\nu \rho \sigma \lambda}\right)-\frac{1}{2} g_{\mu \nu} R_{\mathrm{GB}}^{2}, \\
P_{\mu \nu \rho \sigma} & =R_{\mu \nu \rho \sigma}+2 g_{\mu[\sigma} R_{\rho] \nu}+2 g_{\nu[\rho} R_{\sigma] \mu}+R g_{\mu[\rho} g_{\sigma] \nu} .
\end{aligned}
$$

In the above relations, $P_{\mu \nu \rho \sigma}$ is the divergence free part of the Riemann tensor, i.e., $\nabla_{\mu} P_{\nu \rho \sigma}^{\mu}=0$. One notes that formally, the Eqs. (3) can be written in an Einstein-like form as

$$
G_{\mu \nu}=\frac{1}{2} T_{\mu \nu}^{(\mathrm{eff})},
$$

with an effective energy-momentum tensor acquiring a contribution due to the GBd term

$$
T_{\mu \nu}^{(\mathrm{eff})}=T_{\mu \nu}^{(\phi)}-2 \alpha e^{-\gamma \phi} T_{\mu \nu}^{(\mathrm{GBd})},
$$

where

$$
T_{\mu \nu}^{(\mathrm{GBd})}=H_{\mu \nu}+4\left(\gamma^{2} \nabla^{\rho} \phi \nabla^{\sigma} \phi-\gamma \nabla^{\rho} \nabla^{\sigma} \phi\right) P_{\mu \rho \nu \sigma} .
$$

Variation of Eq. (1) with respect to the scalar field leads to a generalized Klein-Gordon equation,

$$
\nabla^{2} \phi-\alpha \gamma e^{-\gamma \phi} R_{\mathrm{GB}}^{2}=0 .
$$

\section{B. The Ansatz and equations of motion}

We are interested in stationary, axially symmetric spacetimes possessing two commuting Killing vector fields, $\xi$ and $\eta$, with

$$
\xi=\partial_{t}, \quad \text { and } \quad \eta=\partial_{\varphi},
$$

in a system of adapted coordinates. Such spacetimes are usually described by a Lewis-Papapetrou-type Ansatz [25], which satisfies the circularity condition and contains four unknown functions. In this work we shall use a version of this Ansatz as originally introduced in [26], with a line element parametrization

$$
\begin{aligned}
d s^{2}= & -f d t^{2}+\frac{m}{f}\left(d r^{2}+r^{2} d \theta^{2}\right) \\
& +\frac{l}{f} r^{2} \sin ^{2} \theta\left(d \varphi-\frac{\omega}{r} d t\right)^{2},
\end{aligned}
$$

with $r, \theta, \varphi$ "quasi-isotropic" spherical coordinates and $t$ the time coordinate. Also, $f, m, l$, and $\omega$ are functions of $r$ and $\theta$. The scalar field is also a function of the coordinates $r$ and $\theta$ only,

$$
\phi=\phi(r, \theta) .
$$

\section{Boundary conditions and asymptotic behavior}

\section{Large $r$ asymptotics}

The solutions in this work approach a Minkowski spacetime background as $r \rightarrow \infty$. This implies the following boundary conditions [27]

$$
\left.f\right|_{r=\infty}=\left.m\right|_{r=\infty}=\left.l\right|_{r=\infty}=1,\left.\quad \omega\right|_{r=\infty}=\left.\phi\right|_{r=\infty}=0 .
$$


Since the scalar field is massless, one can construct an approximate solution of the field equations (3), (8) compatible with these asymptotics as a power series in $1 / r$. The leading order terms of such an expansion are

$$
\begin{aligned}
& f=1-\frac{2 M}{r}+\frac{2 M^{2}}{r^{2}}+\left(\frac{1}{3} M\left(C_{1}-4 M^{2}\right)-2 M_{2} P_{2}(\cos \theta)\right) \frac{1}{r^{3}}+O\left(\frac{1}{r^{4}}\right), \\
& m=1+\frac{C_{1}}{r^{2}}-\frac{M^{2}+2 C_{1}+D^{2} / 4}{r^{2}} \sin ^{2} \theta+O\left(\frac{1}{r^{3}}\right), \\
& l=1+\frac{C_{1}}{r^{2}}+O\left(\frac{1}{r^{3}}\right), \quad \omega=-\frac{2 a M}{r^{2}}+\frac{6 a M^{2}}{r^{3}}+O\left(\frac{1}{r^{4}}\right), \quad \phi=-\frac{D}{r}+O\left(\frac{1}{r^{3}}\right),
\end{aligned}
$$

where $M, C_{1}, a, M_{1}$, and $D$ are free parameters, while $P_{2}(\cos \theta)$ is a Legendre polynomial of 2 nd degree.

\section{Expansion on the event horizon}

The event horizon of these stationary black hole solutions resides at a surface of constant radial coordinate, $r=r_{\mathrm{H}}>0$, and is characterized by the condition $f\left(r_{\mathrm{H}}\right)=0$ [26]. At a regular horizon the metric functions must satisfy

$$
\left.f\right|_{r=r_{\mathrm{H}}}=\left.m\right|_{r=r_{\mathrm{H}}}=\left.l\right|_{r=r_{\mathrm{H}}}=0,\left.\quad \omega\right|_{r=r_{\mathrm{H}}}=\omega_{\mathrm{H}},
$$

where $\omega_{\mathrm{H}}$ is a constant, while the condition imposed on the scalar field is

$$
\left.\partial_{r} \phi\right|_{r=r_{\mathrm{H}}}=0 .
$$

Again, it is possible to construct an approximate (power series) solution, this time in terms of

$$
\delta=\frac{r}{r_{\mathrm{H}}}-1 .
$$

For nonextremal solutions (the case considered explicitly in this work), the first terms in the near horizon expansion read

$$
\begin{aligned}
f(r, \theta) & =\delta^{2} f_{2}(\theta)(1-\delta)+O\left(\delta^{4}\right), \quad m(r, \theta)=\delta^{2} m_{2}(\theta)(1-3 \delta)+O\left(\delta^{4}\right), \\
l(r, \theta) & =\delta^{2} l_{2}(\theta)(1-3 \delta)+O\left(\delta^{4}\right), \quad \omega(r, \theta)=\omega_{\mathrm{H}}(1+\delta)+O\left(\delta^{2}\right), \quad \phi(r, \theta)=\phi_{0}(\theta)+O\left(\delta^{2}\right),
\end{aligned}
$$

with $f_{2}, m_{2}, l_{2}$, and $\phi_{0}$ unspecified functions [28] and $\omega_{\mathrm{H}}$ a constant.

\section{Behavior on the symmetry axis}

The conditions of axial symmetry and regularity impose the following boundary conditions on the symmetry axis, i.e., at $\theta=0, \pi$ :

$\left.\partial_{\theta} f\right|_{\theta=0, \pi}=\left.\partial_{\theta} m\right|_{\theta=0, \pi}=\left.\partial_{\theta} l\right|_{\theta=0, \pi}=\left.\partial_{\theta} \omega\right|_{\theta=0, \pi}=0$, for the metric function, while for the scalar field one imposes

$$
\left.\partial_{\theta} \phi\right|_{\theta=0, \pi}=0 .
$$

Again, it is possible to construct an approximate form of the solutions near the symmetry axis as a power series in $\theta$ (and $\pi-\theta$, respectively). For example, the first terms in such an expansion as $\theta \rightarrow 0$ reads [29]

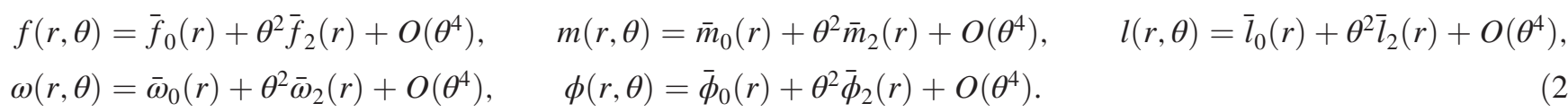

Also, all solutions discussed in this work are symmetric with respect to a reflection on the equatorial plane, $\theta=\pi / 2$. Therefore, in the numerical calculations, it is sufficient to consider the range $0 \leq \theta \leq \pi / 2$ for the angular variable $\theta$. Then the metric functions and the scalar field are required to satisfy Neuman boundary conditions in the equatorial plane,

$$
\begin{aligned}
\left.\partial_{\theta} f\right|_{\theta=\pi / 2} & =\left.\partial_{\theta} m\right|_{\theta=\pi / 2}=\left.\partial_{\theta} l\right|_{\theta=\pi / 2}=\left.\partial_{\theta} \omega\right|_{\theta=\pi / 2} \\
& =\left.\partial_{\theta} \phi\right|_{\theta=\pi / 2}=0 .
\end{aligned}
$$

\section{General relations and quantities of interest}

Starting with the horizon properties, we note that the solutions possess an event horizon of spherical 
topology, the metric of a spatial cross-section of the horizon being

$d \Sigma^{2}=h_{i j} d x^{i} d x^{j}=r_{\mathrm{H}}^{2}\left(\frac{m_{2}(\theta)}{f_{2}(\theta)} d \theta^{2}+\frac{l_{2}(\theta)}{f_{2}(\theta)} \sin ^{2} \theta d \varphi^{2}\right)$.

The Killing vector field

$$
\chi=\partial_{t}-\frac{\omega_{\mathrm{H}}}{r_{\mathrm{H}}} \partial_{\varphi}
$$

is orthogonal to and null on the horizon [25]. The parameter $\omega_{\mathrm{H}}$ which enters the event horizon boundary conditions (14) fixes the event horizon angular velocity $\Omega_{\mathrm{H}}$ of the BHs,

$$
\Omega_{\mathrm{H}}=-\frac{\xi^{2}}{\xi \cdot \eta}=-\left.\frac{g_{\varphi t}}{g_{t t}}\right|_{r_{\mathrm{H}}}=\frac{\omega_{\mathrm{H}}}{r_{\mathrm{H}}}
$$

As usual, we introduce the Hawking temperature $T_{\mathrm{H}}=\kappa /(2 \pi)$, where $\kappa$ is the surface gravity defined as $\kappa^{2}=-\left.\frac{1}{2}\left(\nabla_{a} \chi_{b}\right)\left(\nabla^{a} \chi^{b}\right)\right|_{r_{\mathrm{H}}}$, which yields

$$
T_{\mathrm{H}}=\frac{1}{2 \pi r_{\mathrm{H}}} \frac{f_{2}(\theta)}{\sqrt{m_{2}(\theta)}}
$$

[we recall $m_{2}(\theta), m_{2}(\theta)$ and $l_{2}(\theta)$ are functions which enter the near horizon expansion (17)].

The event horizon area of the BHs is given by

$$
A_{\mathrm{H}}=2 \pi r_{\mathrm{H}}^{2} \int_{0}^{\pi} d \theta \frac{\sqrt{l_{2}(\theta) m_{2}(\theta)}}{f_{2}(\theta)} .
$$

The Einstein gravity BHs possess an entropy which is a quarter of the event horizon area. However, the entropy of the EGBd BHs acquires an extra contribution which is induced by the GB term in the action. Then the total entropy can be written in Wald's form [30] as an integral over the event horizon

$$
S=\frac{1}{4} \int_{\Sigma_{\mathrm{H}}} d^{2} x \sqrt{h}\left(1+2 \alpha e^{-\gamma \phi} \tilde{R}\right),
$$

where $h$ is the determinant of the induced metric on the horizon [as given by (22)], and $\tilde{R}$ is the event horizon curvature. Its explicit form for the Ansatz in this work reads

$$
\begin{aligned}
S= & S_{\mathrm{E}}+S_{\mathrm{GBd}}, \quad \text { with } \quad S_{\mathrm{E}}=\frac{1}{2} \pi r_{\mathrm{H}}^{2} \int_{0}^{\pi} d \theta \frac{\sqrt{l_{2} m_{2}}}{f_{2}}, \quad \text { and } \\
S_{\mathrm{GBd}}= & \frac{1}{2} \pi \alpha \int_{0}^{\pi} d \theta \frac{e^{-\gamma \phi_{0}}}{l_{2}^{3 / 2} m_{2}^{5 / 2}}\left(2 m_{2}^{2} l_{2}^{\prime 2} \sin \theta-m_{2} l_{2}\left(-3 \sin \theta l_{2}^{\prime} m_{2}^{\prime}+4 m_{2}\left(2 \cos \theta l_{2}^{\prime}+\sin \theta l_{2}^{\prime \prime}\right)\right)\right. \\
& \left.+l_{2}^{2}\left(8 m_{2}^{2} \sin \theta-3 \sin \theta m_{2}^{\prime 2}+2 m_{2}\left(3 \cos \theta m_{2}^{\prime}+\sin \theta m_{2}^{\prime \prime}\right)\right)\right),
\end{aligned}
$$

with a prime denoting a derivative with respect to $\theta$.

Similar to GR solutions, the total mass $M$ and the angular momentum $J$ are read from the asymptotic behavior of the metric functions:

$$
\begin{aligned}
& g_{t t}=-f+\frac{l w^{2}}{f} \sin ^{2} \theta=-1+\frac{2 M}{r}+\cdots, \\
& g_{\varphi t}=-\frac{l w r}{f} \sin ^{2} \theta=-\frac{2 J}{r} \sin ^{2} \theta+\cdots .
\end{aligned}
$$

Moreover, the solutions possess a dilaton charge $D$, which is read from the first term in the far field asymptotics of the scalar field as given in (13).

Remarkably, as usual in (asymptotically flat, pure Einstein) BH mechanics, the temperature, entropy, and the global charges are related through a Smarr mass formula, which reads

$$
M=2 T_{\mathrm{H}} S+2 \Omega_{\mathrm{H}} J-\frac{D}{2 \gamma} .
$$

This Smarr relation is obtained by starting from the usual Komar-like expressions, and making use of the equations of motion, and the approximate form of the solution at the horizon, on the symmetry axis and at infinity [31]. The EGBd BHs satisfy also the first law

$$
d M=T_{\mathrm{H}} d S+\Omega_{\mathrm{H}} d J
$$

\section{E. The numerical approach}

The functions $\mathcal{F}_{i}=(f, m, l, \omega ; \phi) \quad(i=1, \ldots, 4)$ are solutions of a complicated set of partial differential equations which are found by plugging the Ansatz (10), (11) into the field equations (3), (8). Starting with the generalized Klein-Gordon equation (8), we note that this reduces to a complicated relation involving first and second derivatives of all functions $\mathcal{F}_{i}$. (This equation is too complicated to display here.) Second, concerning the generalized Einstein equations (3) for the metric potentials, the only nontrivial components are $E_{t}^{t}, E_{r}^{r}, E_{\theta}^{\theta}, E_{\varphi}^{\varphi}, E_{\varphi}^{t}$, and $E_{r}^{\theta}$. Following the scheme originally proposed in [26] (for 
solutions of Einstein gravity coupled with non-Abelian fields), these six equations are divided into two groups. First, four of them are solved together with the scalar field equation (8). This yields a coupled system of five partial differential equations on the five unknown functions $\mathcal{F}_{i}$ which is solved in practice. The four equations for the metric functions are a suitable linear combination of $E_{t}^{t}, E_{\varphi}^{\varphi}, E_{\varphi}^{t}$, and $E_{r}^{r}+E_{\theta}^{\theta}$ which diagonalizes [32] the corresponding Einstein tensor parts with respect to $\hat{O} f$, $\hat{O} l$, $\hat{O} m$, and $\hat{O} \omega$, respectively (with the operator $\hat{O}=\partial_{r r}+\frac{1}{r^{2}} \partial_{\theta \theta}$ ). The remaining two equations $E_{r}^{r}-E_{\theta}^{\theta}$ and $E_{r}^{\theta}$ are treated as constraints and used to check the accuracy of the numerical method. Again, the explicit form of the generalized Einstein equations is too complicated to display here, each equation containing around 340 independent terms.

Restricting the domain of integration to the region outside the horizon, in the next step one introduces a new radial variable $x=1-r_{\mathrm{H}} / r$ which maps the semiinfinite region $\left[r_{\mathrm{H}}, \infty\right)$ to the closed region $[0,1]$. This leads to the following substitutions in the differential equations $r \mathcal{F}_{, r} \rightarrow \frac{1}{r_{\mathrm{H}}}(1-x) \mathcal{F}_{, x} \quad$ and $\quad r^{2} \mathcal{F}_{, r r} \rightarrow \frac{1}{r_{\mathrm{H}}^{2}}\left((1-x)^{2} \mathcal{F}_{, x x}-\right.$ $\left.2(1-x) \mathcal{F}_{, x}\right)$ for each function $\mathcal{F}_{i}$.

The equations for $\mathcal{F}_{i}$ are then discretized on a nonequidistant grid in $x$ and $\theta$. Typical grids used have sizes $91 \times 51$, covering the integration region $0 \leq x \leq 1$ and $0 \leq \theta \leq \pi / 2$. All numerical calculations are performed by using the professional package FIDISOL/CADSOL [33], which uses a Newton-Raphson method. This code provides also an error estimate for each unknown function. For the solutions in this work, the typical numerical error for the functions is estimated to be lower than $10^{-3}$. The Smarr relation (29) and the 1st law (30) provide further tests of the numerical accuracy, leading to error estimates of the same order.

In this approach, one provides three input parameters: $\alpha, r_{\mathrm{H}}$ and $\Omega_{\mathrm{H}}=\frac{\omega_{\mathrm{H}}}{r_{\mathrm{H}}}$. The functions $\mathcal{F}_{i}$ are subject to the boundary conditions introduced in Sec. II. The quantities of interest are then computed from the numerical output. [For example, the mass $M$, and the angular momentum $J$ are extracted from the asymptotic expressions (29), while the Hawking temperature, the entropy and the horizon area are obtained from the event horizon data, etc.]

\section{F. Known limits of the model}

Before discussing the properties of the EGBd spinning $\mathrm{BHs}$, it is useful to briefly review the properties of the solutions in two important limits of the general model.

\section{Kerr metric: the $\alpha=0$ solutions}

The first case is found for $\alpha=0$, i.e., an Einstein(massless) scalar field theory. Then, as implied by classical no-hair theorems (see, e.g., the recent review [34]), the scalar field vanishes, $\phi=0$, and the only physically relevant spinning solution is the Kerr BH. This metric is given usually in Boyer-Lindquist coordinates; however, it can also be written by employing the metric Ansatz (10) (i.e., a quasi-isotropic coordinate system), with

$$
\begin{aligned}
& f=\left(1-\frac{r_{\mathrm{H}}^{2}}{r^{2}}\right)^{2} \frac{F_{1}}{F_{2}}, \quad l=\left(1-\frac{r_{\mathrm{H}}^{2}}{r^{2}}\right)^{2}, \\
& m=\left(1-\frac{r_{\mathrm{H}}^{2}}{r^{2}}\right)^{2} \frac{F_{1}^{2}}{F_{2}}, \quad \omega=\frac{2 M \sqrt{M^{2}-4 r_{\mathrm{H}}^{2}}}{r^{2}} \frac{\left(1+\frac{M}{r}+\frac{r_{\mathrm{H}}^{2}}{r^{2}}\right)}{F_{2}},
\end{aligned}
$$

where

$$
\begin{aligned}
F_{1}= & \frac{2 M^{2}}{r^{2}}+\left(1-\frac{r_{\mathrm{H}}^{2}}{r^{2}}\right)^{2}+\frac{2 M}{r}\left(1+\frac{r_{\mathrm{H}}^{2}}{r^{2}}\right) \\
& -\frac{M^{2}-4 r_{\mathrm{H}}^{2}}{r^{2}} \sin ^{2} \theta, \\
F_{2}= & \left(\frac{2 M^{2}}{r^{2}}+\left(1-\frac{r_{\mathrm{H}}^{2}}{r^{2}}\right)^{2}+\frac{2 M}{r}\left(1+\frac{r_{\mathrm{H}}^{2}}{r^{2}}\right)\right)^{2} \\
& -\left(1-\frac{r_{\mathrm{H}}^{2}}{r^{2}}\right)^{2} \frac{M^{2}-4 r_{\mathrm{H}}^{2}}{r^{2}} \sin ^{2} \theta .
\end{aligned}
$$

The above metric functions contain two parameters, $M$-the $\mathrm{BH}$ mass, and $r_{\mathrm{H}}$ - the event horizon radius, with $M \geq 2 r_{\mathrm{H}}$. To make contact with the numerical approach discussed above, one has to express $M$ as a function of the horizon velocity (which enters the boundary conditions at $\left.r=r_{\mathrm{H}}\right)$. This is done by inverting the relation

$$
\Omega_{\mathrm{H}}=\frac{\sqrt{M^{2}-4 r_{\mathrm{H}}^{2}}}{2 M\left(M+2 r_{\mathrm{H}}\right)} .
$$

Then, one finds that given a fixed value of $\Omega_{\mathrm{H}}$, the Kerr $\mathrm{BH}$ (written in quasi-isotropic coordinates) exhibits two branches of solutions. The first one starts with the Minkowski spacetime, and extends up to a maximal value of $r_{\mathrm{H}}$, where a second branch emerges and tends backwards towards $r_{\mathrm{H}}=0$. The maximal value of $r_{\mathrm{H}}$ depends on $\Omega_{\mathrm{H}}$, with $r_{\mathrm{H}}^{(\max )}=\frac{1}{2 \Omega_{\mathrm{H}}} \frac{\sqrt{\frac{2}{1+\sqrt{5}}}}{3+\sqrt{5}}$. As $r_{\mathrm{H}} \rightarrow 0$ on this second branch, an extremal $\mathrm{BH}$ is approached, which saturates the Kerr bound, $J=M^{2}$.

The entropy, angular momentum and the Hawking temperature of the Kerr solution are given by

$$
\begin{aligned}
S & =2 \pi M\left(M+2 r_{\mathrm{H}}\right), \quad J=M \sqrt{M^{2}-4 r_{\mathrm{H}}^{2}}, \\
T_{\mathrm{H}} & =\frac{1}{4 \pi M} \frac{1}{1+\frac{M}{2 r_{\mathrm{H}}}},
\end{aligned}
$$

respectively. 


\section{Spherically symmetric black holes in EGBd theory}

Another important limit is found by taking the static limit of the general model with $\alpha \neq 0$. The solutions in this case are spherically symmetric, and have been discussed in Refs. [8-13]. All these papers employ in their study Schwarzschild-like coordinates. However, the solutions can also be studied by employing the metric Ansatz (10), with $f(r, \theta)=f(r), \quad l(r, \theta)=m(r, \theta)=m(r)$, $\omega(r, \theta)=0$ and $\phi(r, \theta)=\phi(r)$. Thus the corresponding Ansatz in isotropic coordinates reads

$$
\begin{aligned}
d s^{2} & =\frac{m(r)}{f(r)}\left(d r^{2}+r^{2}\left(d \theta^{2}+\sin ^{2} \theta d \varphi^{2}\right)\right)-f(r) d t^{2} \quad \text { and } \\
\phi & =\phi(r) .
\end{aligned}
$$

The metric functions $f(r), m(r)$ and the scalar field $\phi(r)$ can be found by solving a system of ordinary differential equations.

The solutions possess a relatively simple near horizon expansion with (we recall $\delta=r / r_{\mathrm{H}}-1$ )

$$
\begin{aligned}
& f(r)=f_{2} \delta^{2}(1-\delta)+\cdots, \quad m(r)=m_{2} \delta^{2}(1-3 \delta)+\cdots, \\
& \phi(r)=\phi_{0}+\phi_{2} \delta^{2}+\cdots,
\end{aligned}
$$

with $f_{2}, m_{2}$, and $\phi_{0}$ parameters fixed by the numerical calculations, and

$$
\phi_{2}=\frac{e^{3 \gamma \phi_{0}} m_{2}^{3} r_{\mathrm{H}}^{4}}{768 \alpha^{3} f_{2}^{3} \gamma^{3}}\left(1-\sqrt{1-\frac{96 e^{-2 \gamma \phi_{0}} \alpha^{2} f_{2}^{2} \gamma^{2}}{m_{2}^{2} r_{\mathrm{H}}^{4}}}\right)^{2},
$$

while the corresponding expressions for $r \rightarrow \infty$ can be read from (13).

An interesting feature of these BHs is the existence, for a given $\alpha$, of a minimal horizon size of the solutions [10]. This follows from the "reality" condition as imposed by the expression (36) for $\phi_{2}$,

$$
1-\frac{96 e^{-2 \gamma \phi_{0}} \alpha^{2} f_{2}^{2} \gamma^{2}}{m_{2}^{2} r_{\mathrm{H}}^{4}}>0
$$

which can also be written as

$$
A_{\mathrm{H}}>16 \pi \sqrt{6} \alpha \gamma e^{-\gamma \phi_{0}}
$$

At $r_{\mathrm{H}}^{(c r)}$ the solution becomes singular [11-13].

A close inspection of the solutions and their properties reveals that very near to the critical minimal value of the horizon radius $r_{\mathrm{H}}^{(c r)}$ a minuscule second branch of $\mathrm{BH}$ solutions can arise [11-13]. Along this branch the mass then increases with decreasing horizon radius. For $\gamma=1$ it covers the range

$$
2.4053 \leq \frac{M}{\sqrt{\alpha}} \lesssim 2.4055 \text {. }
$$

The existence of this minuscule second branch depends on the value of $\gamma$ [13], e.g., for $\gamma=1 / 2$ is it no longer present.

Consequently, as discussed in [10-14], a lower bound is imposed on the $\mathrm{BH}$ mass, which for $\gamma=1$ reads [35]

$$
0 \leq \frac{\alpha}{M^{2}} \lesssim 0.1728
$$

Then for a given mass, the static EGBd BHs with $\gamma=1$ exist for a limited range of the entropy, of the area and of the temperature, with

$4 \pi \leq \frac{S}{M^{2}} \lesssim 15.082, \quad 16 \pi \leq \frac{A_{\mathrm{H}}}{M^{2}} \lesssim 42.842 \quad$ and

$\frac{1}{8 \pi} \leq T_{\mathrm{H}} M \lesssim 0.043$.

Finally let us mention that the scalar charge $D$ is not an independent quantity, but depends on the BH mass. Thus the scalar hair is of secondary type.

\section{THE PROPERTIES OF EGBD SPINNING BLACK HOLES}

\section{A. Constructing the solutions and limits}

We start by recalling that all solutions in this work have a fixed value $\gamma=1$ of the dilaton coupling constant.

As expected, all spherically symmetric EGBd BHs discussed above possess rotating generalizations. In principle, they can be constructed in closed form by considering a (double) perturbative approach in terms of the dimensionless parameters $\alpha / M^{2}$ and $J / M^{2}$, see, e.g., [15].

The nonperturbative solutions are found by directly solving the EGBd equations for the functions $\mathcal{F}_{i}$ without any approximation. For all solutions we have found, the metric functions $F_{i}$ (and their first and second derivatives with respect to $r$ and $\theta$ ) have smooth profiles, which leads to finite curvature invariants on the full domain of integration, in particular on the event horizon. The shape of the functions $f, l, m$, and $\omega$ is rather similar to the GR case.

To illustrate these features, we display in Figs. 1 and 2 the profile functions of a typical solution together with the Ricci and the Gauss-Bonnet scalars. There, the left column shows 3D plots, whereas the right column displays 2D plots of the corresponding functions in terms of the radial variable for three different values of the angular coordinate; the axes for the 3D plots are $\rho=r \sin \theta$ and $z=r \cos \theta$ (with $r \geq r_{\mathrm{H}}$ ). For example, from Fig. 2 (top panel) one can see that the scalar field possesses a rather complicated shape, with a nontrivial dependence on the angular coordinate $\theta$. Also, as seen in Fig. 2, both $R$ and $R_{\mathrm{GB}}^{2}$ stay finite everywhere, in particular at the horizon. 

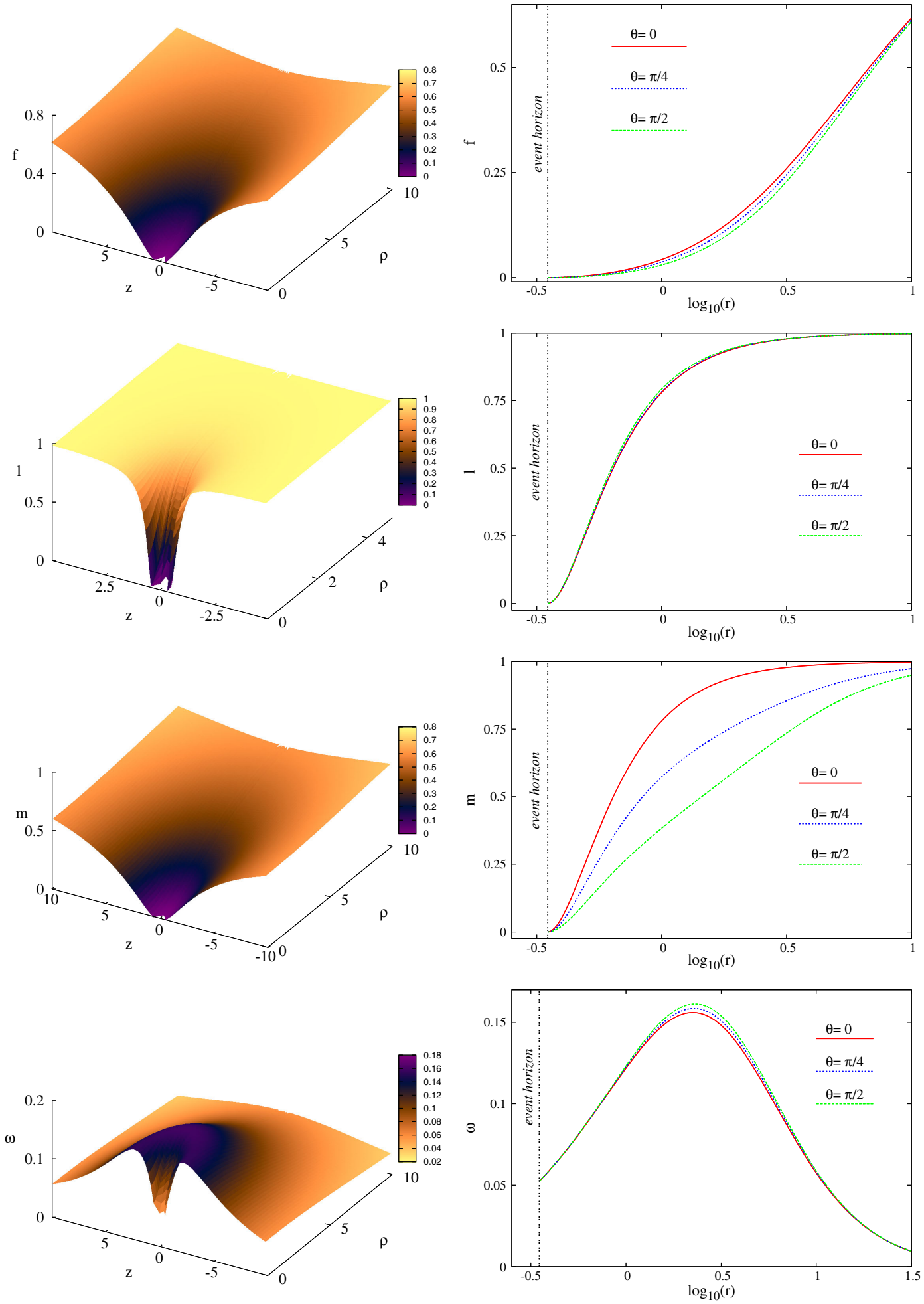

FIG. 1. The metric functions $f, l, m$, and $\omega$ are shown as for a typical EGBd spinning black hole with the input parameters $r_{\mathrm{H}}=0.35$, $\Omega_{\mathrm{H}}=0.15$, and $\alpha=0.5$. This solution has $M=2.472, J=5.886, D=0.231, T_{\mathrm{H}}=.00737, A_{\mathrm{H}}=193.401$, and $S=55.751$.

In our approach, the solutions are computed for fixed sets of $\Omega_{\mathrm{H}}$ and $\alpha$, while the (quasi-isotropic) horizon radius $r_{\mathrm{H}}$ and consequently the event horizon area $A_{\mathrm{H}}$ is varied [36]. As in the Kerr case, discussed above, we typically find two branches of solutions [37] in terms of the quasi-isotropic horizon radius $r_{\mathrm{H}}$. These two branches then merge and end at a maximal value of $r_{\mathrm{H}}$ which depends on $\Omega_{\mathrm{H}}$ and $\alpha$. However, whereas in the Kerr case, the first branch starts 

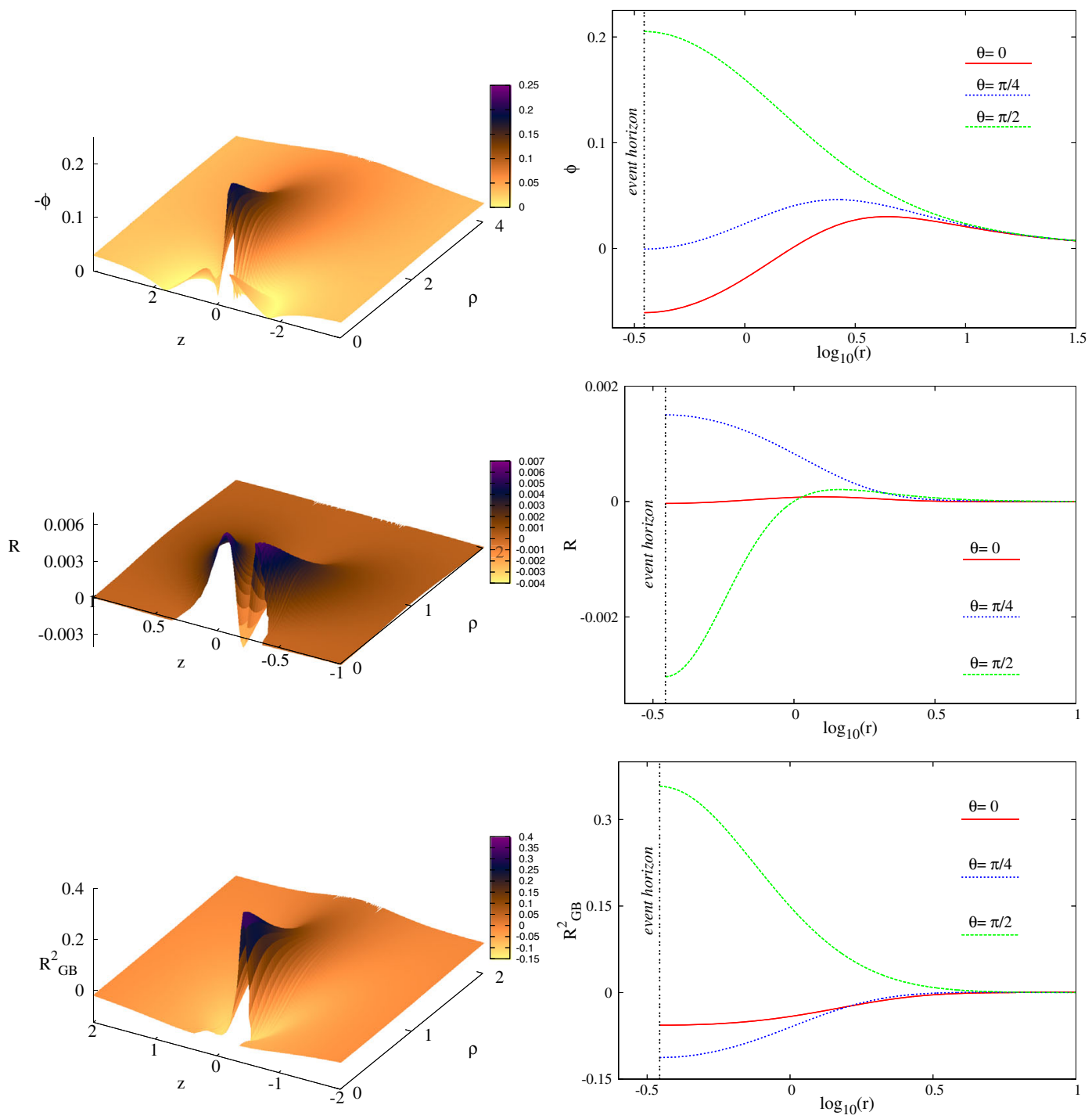

FIG. 2. The scalar field $\phi$ is shown together with the scalar curvature $R$ and the Gauss-Bonnet term (2) for the same solution as in Fig. 1.

with the Minkowski spacetime, in the EGBd case the horizon area cannot shrink to zero. Indeed, similar to the static case, the rotating $\mathrm{BH}$ solutions on the first branch cease to exist for $r_{\mathrm{H}}$ smaller than some critical value $r_{\mathrm{H}}^{(\mathrm{cr})}\left(\alpha, \Omega_{\mathrm{H}}\right)$. We note, that as $r_{\mathrm{H}} \rightarrow r_{\mathrm{H}}^{(\mathrm{cr})}$, the numerical integration becomes delicate. The "technical" reason which causes the solutions to cease to exist beyond $r_{\mathrm{H}}^{(\mathrm{cr})}$ corresponds to a more complicated version of the one found in the static case. It is discussed in Appendix A, and again based on the analysis of the field equations at the event horizon.

\section{B. The domain of existence}

Returning to the discussion of the sets of solutions for $\Omega_{\mathrm{H}}$ and $\alpha$, let us now address the limiting behavior on the second branch. As discussed above, the family of Kerr BHs with fixed $\Omega_{\mathrm{H}}$ ends in an extremal configuration with a novanishing horizon area, which is approached as $r_{\mathrm{H}} \rightarrow 0$. We have found that this applies also for the families of EGBd BHs with fixed $\Omega_{\mathrm{H}}$ and $\alpha$. The extrapolated numerical data [38] always indicates the existence of a $r_{\mathrm{H}} \rightarrow 0$ limiting configuration with vanishing Hawking temperature and nonvanishing global charges, horizon area and entropy. However, unlike the extremal Kerr solution, the extremal EGBd solutions appear to not be regular. That is, the data found for all limiting solutions found so far show that the dilaton field at the horizon seems to diverge at the poles as $r_{\mathrm{H}} \rightarrow 0$, making the extremal solutions singular. Note, however, that the (quasi-isotropic) metric functions tend to well-defined limiting functions.

This behavior is illustrated in Fig. 3, where we show how the value of the scalar field at the north pole on the horizon $(\theta=0)$ changes with the scaled temperature for two values of the dimensionless parameter $\Omega_{\mathrm{H}} \sqrt{\alpha}$. The curves there start at the corresponding critical solutions and end at 


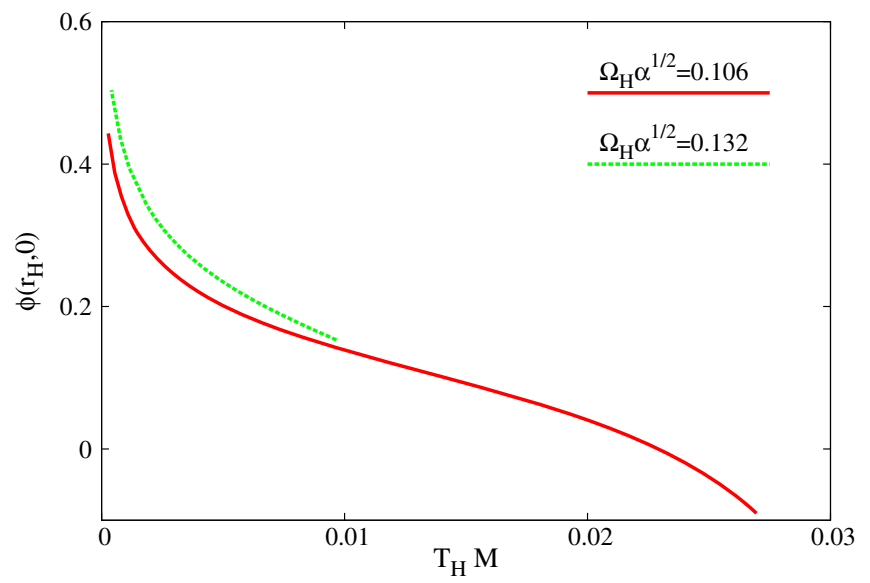

FIG. 3. The value of the scalar field at the poles of the horizon is shown as a function of the scaled temperature for two values of the scaled angular velocity $\Omega_{\mathrm{H}} \sqrt{\alpha}$.

near-extremal ones (beyond which sufficient numerical accuracy is lost). A further argument for the nonexistence of regular extremal solutions is given in Appendix B, and based on an attempt to construct the near-horizon configurations.

Let us now address the domain of existence of the EGBd spinning BHs. As expected, the general pattern is rather complicated, and depends on the value of the parameter $\alpha$, which sets another length scale of the problem, apart from the mass of the solutions $M$.

In Fig. 4 (left panels) the dimensionless horizon area $A_{\mathrm{H}} / M^{2}$, the dimensionless entropy $S / M^{2}$, the dimensionless temperature $T_{\mathrm{H}} M$ and the dimensionless dilaton charge $D / M$ of all solutions are shown as functions of the dimensionless coupling constant $\alpha / M^{2}$. A complementary picture is found when exhibiting the same data as functions of the dimensionless reduced angular momentum $J / M^{2}$, as shown in Fig. 4 (right panels). Note, that in the figure all physical quantities are expressed in units set by the mass of the solutions. The link between these two sets is provided by Fig. 5, where we show the dimensionless reduced angular momentum $J / M^{2}$ as a function of the dimensionless coupling constant $\alpha / M^{2}$. In these plots we include also the lines corresponding to three constant values of the dimensionless parameter $\Omega_{\mathrm{H}} \sqrt{\alpha}$, which provides a measure of how fast the solutions spin.

Also, let us mention that in these plots, the shaded region was obtained by extrapolating to the continuum the results from a set of around one thousand numerical solutions.

In all plots, the region where EGBd spinning BHs exist is delimited by

(i) the set of static EGBd BHs (red solid line);

(ii) the set of extremal (i.e., zero temperature) EGBd BHs (green dashed line), which is found by extrapolating the data for near-extremal configurations;

(iii) the set of critical solutions (blue dotted line);

(iv) and the set of GR solutions-the Kerr BHs (black solid line).
These figures exhibit a number of interesting features of the spinning EGBd solutions:

(a) For a given value of $\alpha$, the lower bound (37) on the $\mathrm{BH}$ mass still holds, irrespective of the value of the angular momentum. Moreover, the solutions saturating this bound have $J=0$.

(b) For a given mass and angular momentum, an EGBd $\mathrm{BH}$ has a higher entropy and temperature than a Kerr $\mathrm{BH}$; however, an EGBd $\mathrm{BH}$ has a lower horizon area than a Kerr BH, except close to the Kerr bound.

(c) The critical static solution has the largest scaled entropy possible for an EGBd BH, while the critical extremal solution has the smallest scaled area. The dilaton charge is also maximal for the critical static solution.

(d) Perhaps the most interesting feature one can notice in Figs. 4 and 5 is that the EGBd spinning BHs can violate the Kerr bound $J / M^{2} \leq 1$, for a particular set of solutions close to extremality. This violation is rather small [39] (with a maximal value of $J / M^{2} \simeq$ 1.03). However, this violation clearly exists, being manifestly above the numerical accuracy of the calculations.

(e) Somehow unexpected, the maximal value of $J / M^{2}$ is found for a value of $\alpha / M^{2} \simeq 0.079$ which is almost in the middle of the allowed interval. At this special point of the domain of existence, where the scaled angular momentum reaches its maximal value, the branches of critical EGBd BHs and singular extremal solutions merge. At the same time, the scaled horizon angular velocity reaches there its maximal value, $\Omega_{\mathrm{H}} \alpha^{1 / 2} \approx 0.135-0.14$.

\section{Further properties}

\section{Horizon and ergoregion}

As mentioned above, similar to the Kerr solution in GR, these EGBd BHs have an event horizon of spherical topology [43]. Geometrically, however, the horizon is a squashed sphere. This can be seen by evaluating the circumference of the horizon along the equator, $L_{e}$, and the circumference of the horizon along the poles, $L_{p}$

$$
L_{e}=\left.2 \pi r_{\mathrm{H}} \sqrt{\frac{l_{2}(\theta)}{f_{2}(\theta)}}\right|_{\theta=\pi / 2}, \quad L_{p}=2 r_{\mathrm{H}} \int_{0}^{\pi} d \theta \sqrt{\frac{m_{2}(\theta)}{f_{2}(\theta)}} .
$$

In Fig. 6 we show the ratio of the equatorial circumference to the polar circumference for several values of the dimensionless quantity $\Omega_{\mathrm{H}} \sqrt{\alpha}$. As expected, the squashing of the horizon produced by the rotation is such that $L_{e} / L_{p}$ is always larger than one.

Further insight into the issue of horizon shape is obtained by considering the isometric embeddings of the horizon in a 

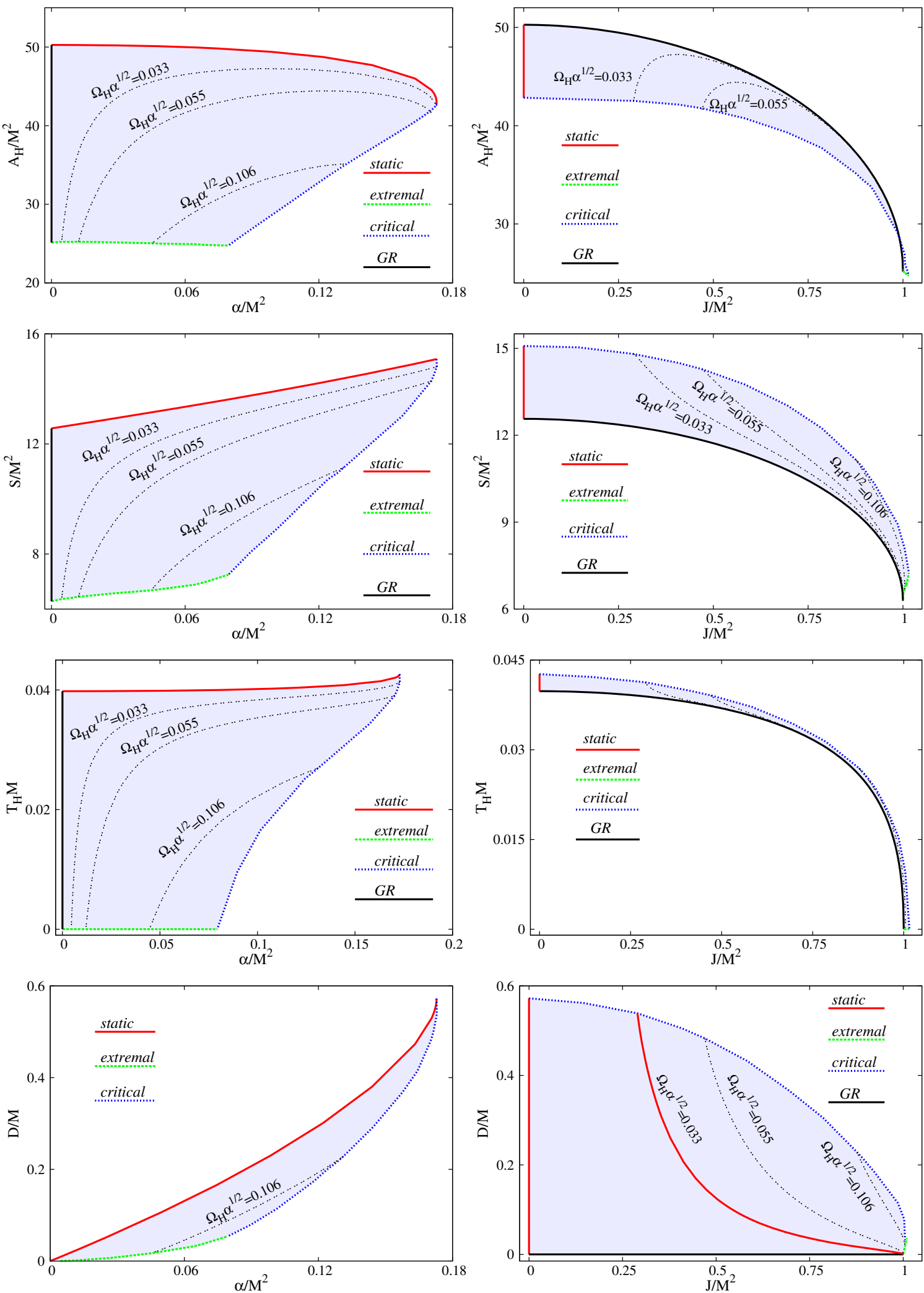

FIG. 4. The domain of existence of the horizon area, entropy, temperature and dilaton charge is shown vs $\alpha$ (left panels) and vs $J$ (right panels). Here and in Fig. 5, all quantities are normalized with respect to the mass of the solutions. 
SPINNING BLACK HOLES IN EINSTEIN-GAUSS- ...

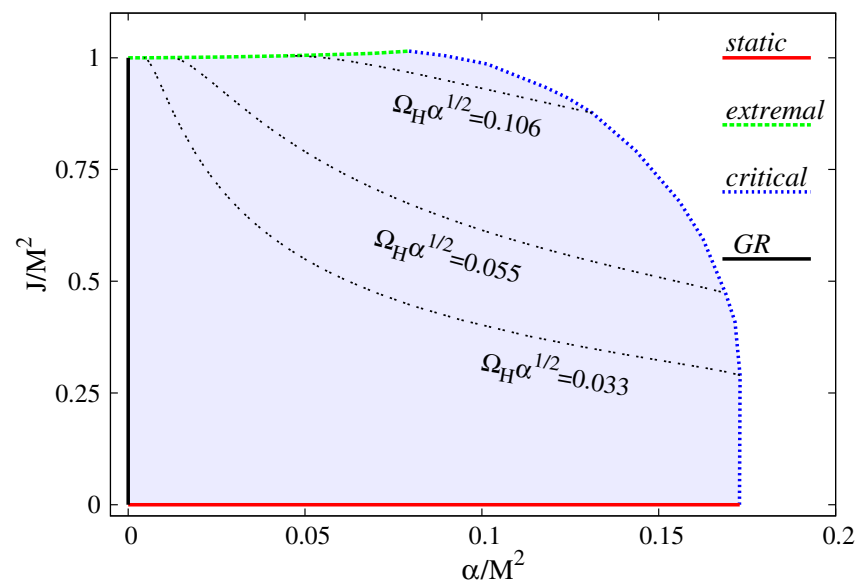

FIG. 5. Domain of existence of EGBd spinning BHs in an angular momentum vs $\alpha$ diagram.

Euclidean space [44]. In Fig. 7 this is done for the solution shown in Figs. 1 and 2 together with a Kerr BH with the same values of the mass and angular momentum. Note that for both GR and EGBd solutions, the embedding of the horizon cannot be performed completely in Euclidean space with the metric $d s^{2}=d x^{2}+d y^{2}+d z^{2}$, and a region (represented by solid lines in that figure) must be embedded in a pseudo-Euclidean space with the metric $d s^{2}=d x^{2}+d y^{2}-d z^{2}$.

On the horizon the scalar field $\phi\left(r_{\mathrm{H}}, \theta\right)$ and the component $T_{t}^{t(\phi)}$ of the scalar field energy-momentum tensor vary with the angular coordinate, as it is manifest in Fig. 8. For example, one can see that $T_{t}^{t(\phi)}$ vanishes both at the poles and in the equatorial plane. Also, the scalar field by itself does not contribute to the angular momentum of these $\mathrm{BHs}$, since $T_{\varphi}^{t(\phi)}=0$ everywhere [45].

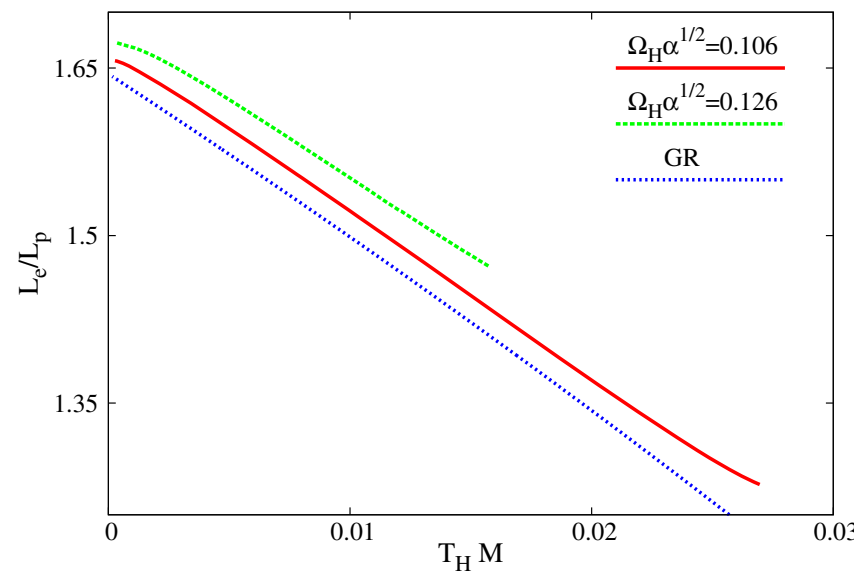

FIG. 6. The ratio $L_{e} / L_{p}$ is shown for two sets of EGBd solutions with fixed values of the dimensionless parameter $\Omega_{\mathrm{H}} \sqrt{\alpha}$, which extend from the critical configurations to the extremal ones. Also shown is this ratio for a set of Kerr solutions close to extremality.
PHYSICAL REVIEW D 93, 044047 (2016)

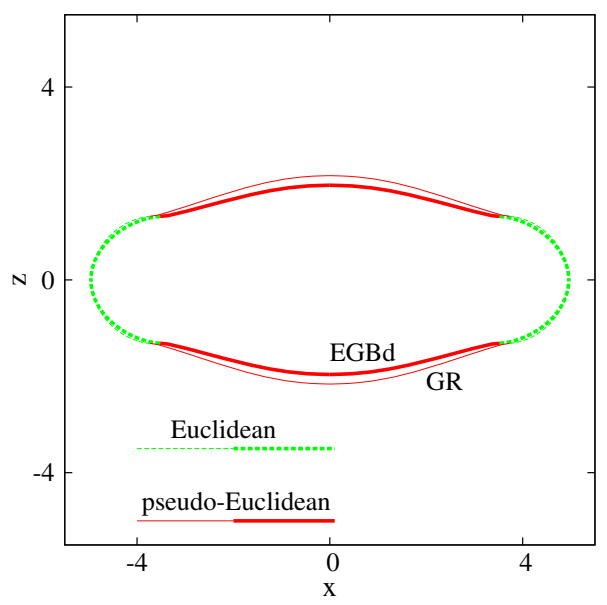

FIG. 7. The shape of the horizon is shown for the EGBd black hole in Figs. 1 and 2 and for a Kerr black hole with the same values of the mass and angular momentum. The solid lines indicate an embedding in pseudo-Euclidean space, the dashed lines in Euclidean space.

We have found that all BHs have an ergoregion, defined as the domain in which $\xi_{\mu} \xi^{\mu}$ is positive (where we recall $\xi=\partial / \partial t$ ). This region is bounded by the event horizon and by the surface(s) where

$$
-f+\sin ^{2} \theta \frac{l}{f} \omega^{2}=0 .
$$

For the Kerr spacetime, this surface has a spherical topology and touches the horizon at the poles. As discussed in [46,47], the ergoregion can be more complicated for BHs with scalar hair, with the possible existence of an additional $S^{1} \times S^{1}$ ergo-surface (ergo-torus). However, we have found that this is not the case for EGBd BHs, where all solutions are Kerr-like and possess a single topologically $S^{2}$ ergosurface [48]. In Fig. 9 (left) we show a cross section of the ergosurface for the (typical) solution exhibited in Figs. 1 and 2. The metric function $g_{t t}$ in the equatorial plane is also shown there (right panel). The event horizon is displayed as the black dotted curve and the ergosurface as the solid red line, while the ergoregion is shaded in blue. Also, $x$ and $z$ are defined as standard Cartesian coordinates in terms of $r$ and $\theta$, which enter (10).

Let us mention also that since the functions $f, l$, and $m$ are always strictly positive outside the horizon, the Lorentzian signature of the metric is preserved there. Moreover, $f>0$ implies the existence of a Cauchy surface and thus the absence of closed timelike curves outside the horizon.

\section{Quadrupole moment and moment of inertia}

On general grounds, one expects the EGBd spinning $\mathrm{BHs}$ to have a number of new phenomenological properties, leading to deviations from the standard Kerr picture in 

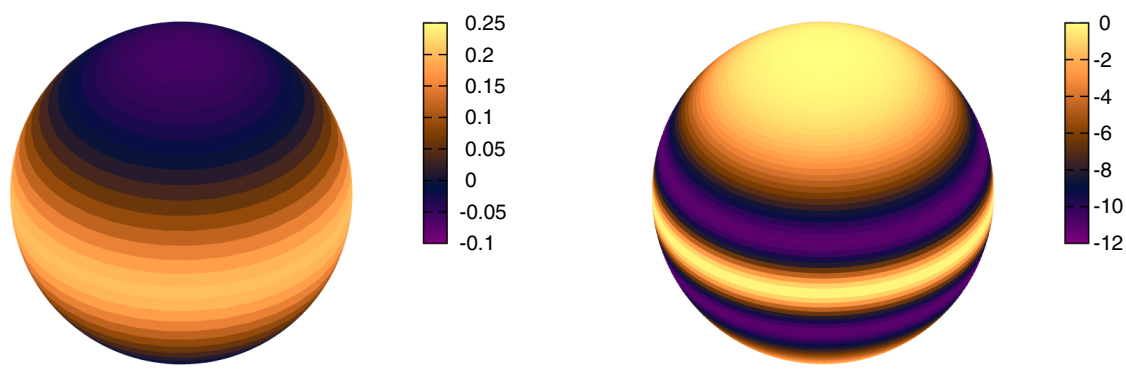

FIG. 8. The scalar field (left panel) and its component $T_{t}^{t(\phi)}$ of the energy momentum tensor (multiplied with a factor of $10^{3}$; right panel) on the horizon, are shown for the typical EGBd spinning solution of Figs. 1 and 2.
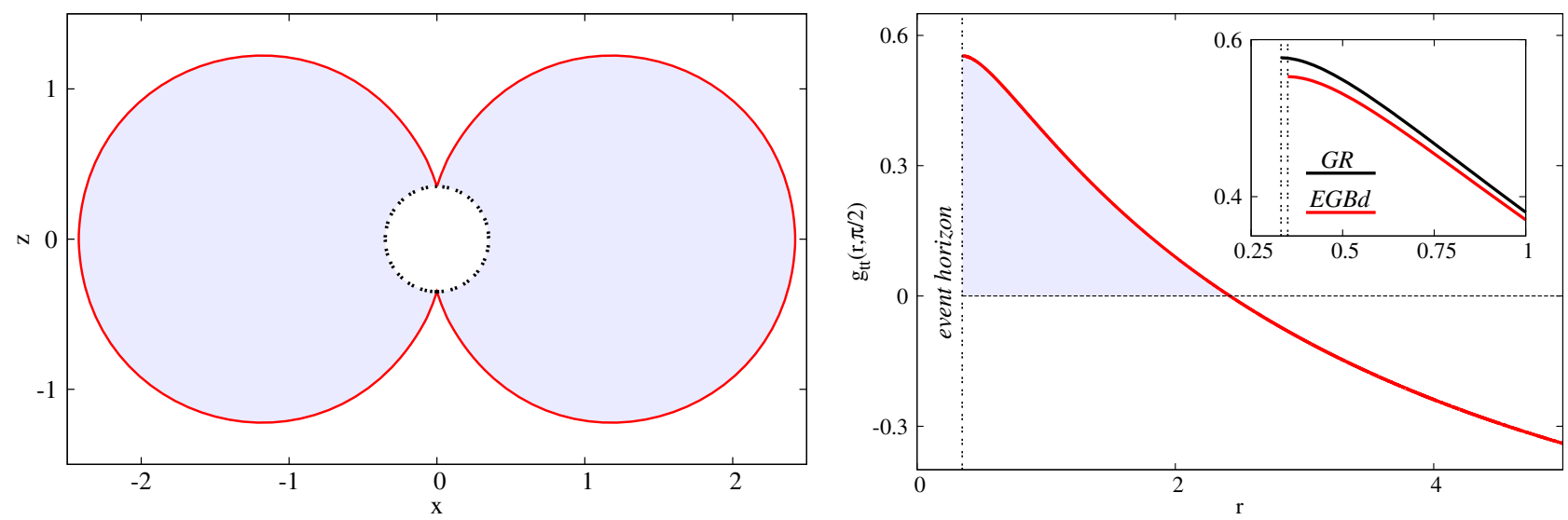

FIG. 9. A cross section of the ergosurface in the $x-z$ plane (left panel) and the metric function $g_{t t}$ in the equatorial plane (right panel) for the EGBd spinning solution in Figs. 1 and 2. In the right panel, the inset shows the near horizon behavior of $g_{t t}(r, \pi / 2)$ for this solution and for a Kerr black hole with the same values of the mass and angular momentum.

GR. Let us start by addressing the issue of the quadrupole moment, $Q$, which is of particular interest since, in principle, it can be measured. Moreover, it can be used to test the no-hair property [50]. As discussed in [20,51], the quadrupole moment of EGBd BHs is encoded in the free constants which enter the large- $r$ expansion of the solutions (13), with [56]

$$
-Q=-M_{2}+\frac{4}{3}\left(\frac{1}{4}+\frac{C_{1}}{2 M^{2}}+\frac{D^{2}}{16 M^{2}}\right) M^{3} .
$$

Since the dilaton field enters the expansion for the quadrupole moment via a Coulomb-like term in (13), $D / r$, and since there is no explicit contribution from the GB term, which decays sufficiently fast, the resulting expression for the quadrupole moment coincides with the analogous term in Einstein-Maxwell theory (after replacing the electromagnetic charge $Q_{e}$ by $D$ ).

In contrast to the EGBd case, the quadrupole moment of the Kerr solution is completely fixed by its global charges, with $Q=-J^{2} / M$. In Fig. 10 the magnitude of the scaled quadrupole moment $Q M / J^{2}$ is exhibited versus the scaled angular momentum $J / M^{2}$. Here the Kerr solution is represented by the line $\left|Q M / J^{2}\right|=1$. The figure shows that the quadrupole moment of EGBd spinning BHs can take considerably different values from the Kerr case. Interestingly, for slow rotation, one finds deviations from the Kerr value of $20 \%$ and more. Moreover, the solutions

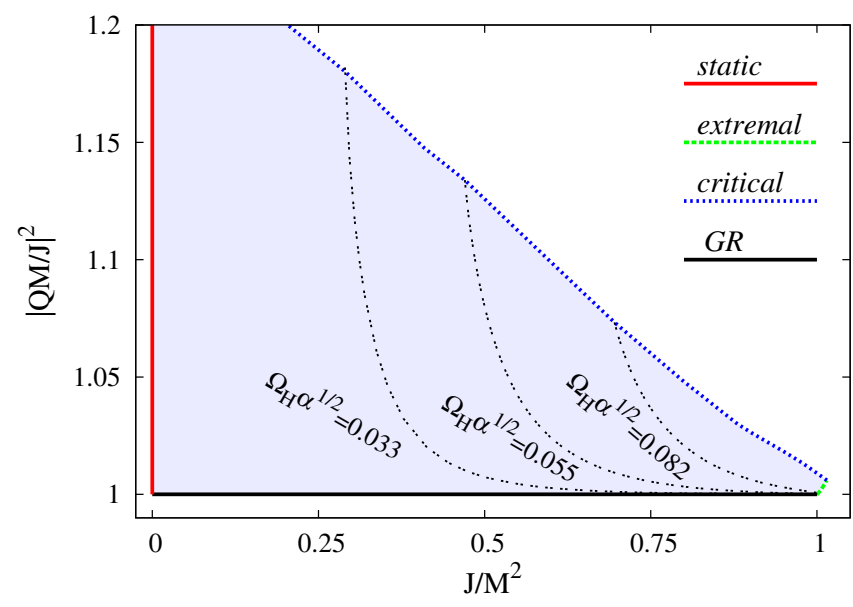

FIG. 10. The magnitude of the scaled quadrupole moment $Q M / J^{2}$ is shown versus the scaled angular momentum $J / M^{2}$ for several values of the scaled horizon angular velocity $\Omega_{\mathrm{H}} \sqrt{\alpha}$. The shaded area represents the domain of existence of the EBGd black holes. 


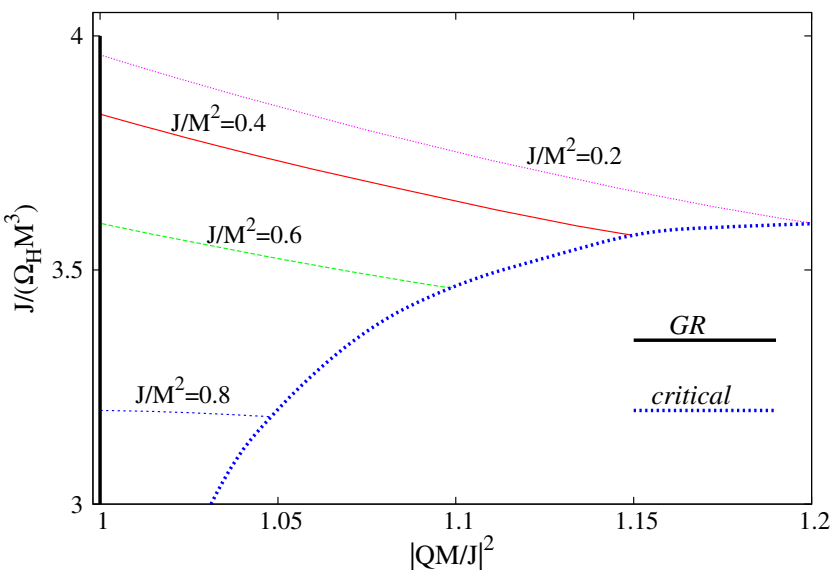

FIG. 11. The scaled moment of inertia $J /\left(\Omega_{\mathrm{H}} M^{3}\right)$ is shown versus the magnitude of the scaled quadrupole moment $Q M / J^{2}$ for several values of the scaled angular momentum $J / M^{2}$.

with $J>M^{2}$, always have a value of the scaled quadrupole moment greater than one.

The scaled moment of inertia $J /\left(\Omega_{\mathrm{H}} M^{3}\right)$ is exhibited in Fig. 11 versus the scaled quadrupole moment. The Kerr values are given by $J /\left(\Omega_{\mathrm{H}} M^{3}\right)=2\left(1+\sqrt{1-j^{2}}\right)$ with $j=J / M^{2}$, and form the vertical line at $\left|Q M / J^{2}\right|=1$, ranging from the value $J /\left(\Omega_{\mathrm{H}} M^{3}\right)=2$ in the extremal rotating case to the value $J /\left(\Omega_{\mathrm{H}} M^{3}\right)=4$, when the static limit $J \rightarrow 0$ is taken.

For the EGBd BHs we have obtained families of solutions with fixed values of the reduced angular momentum $j=J / M^{2}$. For $j \leq 1$ these families of solutions start at their respective Kerr values on the vertical line $\left|Q M / J^{2}\right|=1$, from where they extend, until the respective critical solution is reached. Thus the Kerr values and the critical values again form two of the boundaries of the domain of existence. We have not yet mapped out the full domain of existence, since the limit $j \rightarrow 0$ is difficult to obtain from a numerical point of view, because ratios of small numbers are involved. Here perturbation theory should be helpful, at least in part of the domain. Comparing our results with the perturbative results derived in [17] yields good agreement for small $\alpha$ and small $j$ [20].

\section{Petrov type and ISCOS}

One interesting property of the Kerr metric is that the geodesic equations are separable due to the existence of the Carter constant [57]. This is related to the fact that the Kerr spacetime is Petrov type D. We have investigated the issue of Petrov type for a number of EGBd solutions in different regions of the parameter space and found that all of them are Petrov type I. Thus we expect this to be a generic property of all configurations [58]. Therefore, the existence of a Carter-like constant is highly unlikely in this case.

Clearly, the geodesics of these EGBd BHs are also expected to deviate considerably from those of the Kerr
BHs. The geodesics of static and slowly rotating BHs were considered in [14]. There the dependence of the innermoststable-circular-orbits (ISCOs) on the angular momentum was studied, showing that the ISCO is larger for slowly rotating EGBd BHs than for Kerr BHs, and that the orbital frequency is smaller [14].

While a systematic discussion of the geodesics of the general family of rotating EGBd BHs will be presented elsewhere, we here focus on the special case of ISCOs for the rapidly spinning BHs [18]. The geodesics for circular motion are obtained from the Lagrangian

$$
2 \mathcal{L}=e^{-2 \beta \phi} g_{\mu \nu} \dot{x}^{\mu} \dot{x}^{\nu}=-\epsilon,
$$

where $\epsilon=0$ and 1 massless and massive particles, respectively. The constant $\beta$ fixes the coupling between the matter and the dilaton field. (In heterotic string theory $\beta=0.5$.) Also, a superposed dot denotes the derivative with respect to the affine parameter along the geodesics. As usual, the existence of the Killing vectors $\xi$ and $\eta$ implies the existence of two conserved quantities, the energy $E$ and the angular momentum $L$ of the particle, respectively. Restricting to (timelike) motion in the equatorial plane $(\theta=\pi / 2)$, one finds from (45) the equation

$$
\dot{r}^{2}=e^{2 \beta \phi} \frac{f}{m}\left(e^{2 \beta \phi} \frac{\left(E-\frac{L \omega}{r}\right)^{2}}{f}-e^{2 \beta \phi} \frac{L^{2} f}{r^{2} l}-1\right) \equiv V(r) .
$$

Also, the orbital angular velocity is expressed as

$$
\Omega_{c}=\frac{\dot{\varphi}}{\dot{t}}=\frac{\omega}{r}+\frac{1}{r^{2}} \frac{L f^{2}}{l\left(E-\frac{L \omega}{r}\right)} .
$$

For circular orbits $V(r)=V^{\prime}(r)=0$. This results in two algebraic relations for $E$ and $L$ which are solved analytically. Similar to the Kerr case, this gives two distinct pairs of solutions corresponding to corotating and counterrotating trajectories. Stability of the orbits further requires that the second derivative of the effective potential is negative, $V^{\prime \prime}(r)<0$. This selects a particular value of the radial coordinate $r=r_{\text {ISCO }}$, which separates the stable circular orbits, $r>r_{\text {ISCO }}$, from the unstable ones.

The results for EGBd ISCOs for rapidly rotating BHs are exhibited in Fig. 12 for $\beta=0.5$ together with the corresponding Kerr ISCOs. Note, that we exhibit the circumferential radius $R_{\mathrm{ISCO}}=\left.r \sqrt{m / f}\right|_{r_{\mathrm{ISCO}}}$ here (and not the Boyer-Lindquist coordinate radius). As seen in the figure, the ISCOs of the EBGd BHs remain larger than the Kerr ISCOs (as long as the corresponding Kerr BHs exist), also for rapidly rotating BHs [18]. Finally, we exhibit in Fig. 13 the inverse of the orbital frequencies $\Omega_{c}$ for the EGBd ISCOs for $\beta=0.5$ and for $\beta=0$ and compare again with the Kerr case. For $\beta=0.5$ the frequencies are smaller than 


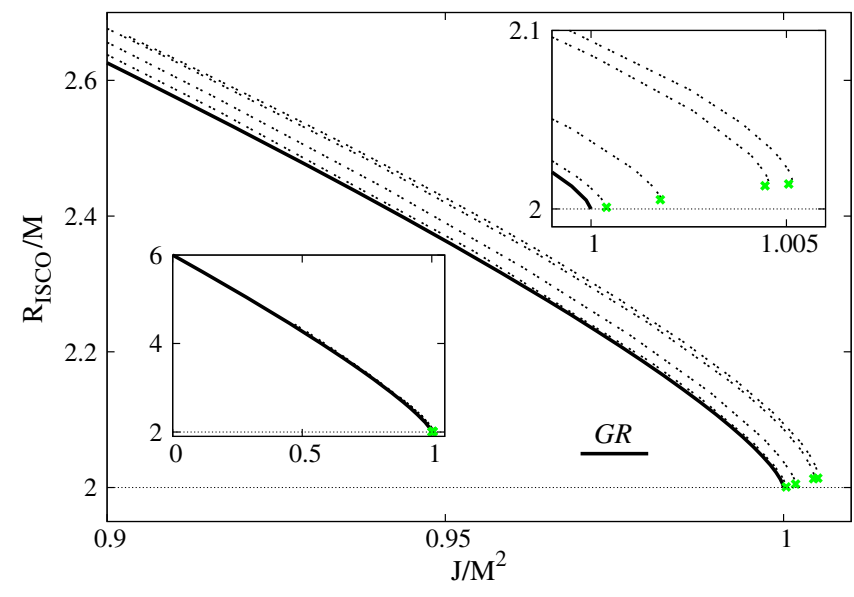

FIG. 12. The circumferential ISCO radius $R_{\text {ISCO }}$ (given in units of the mass) is shown versus the reduced angular momentum $J / M^{2}$ for several values of the scaled event horizon velocity $\Omega_{\mathrm{H}} \sqrt{\alpha}=0.055$ (the curve closest to the GR one), 0.082, 0.106, and 0.11 .

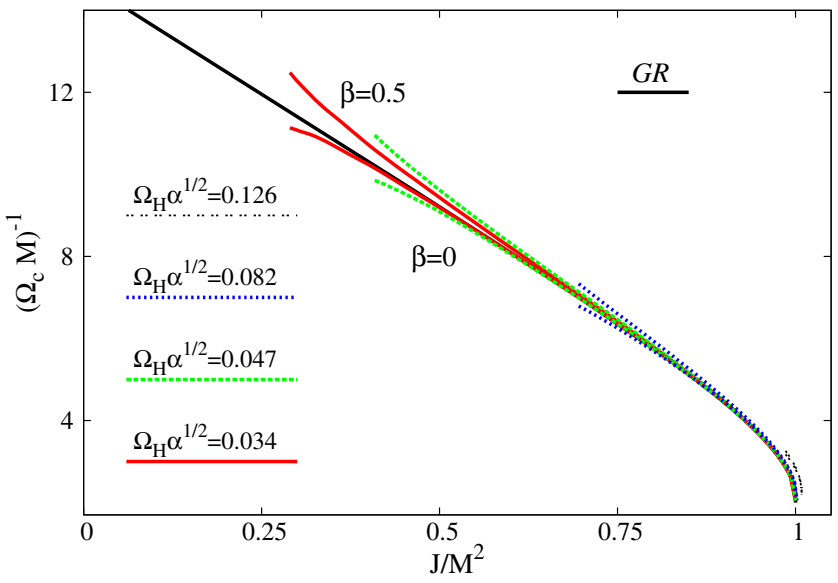

FIG. 13. The inverse of the orbital frequency $\Omega_{c}$ is shown versus the angular momentum for coupling constant $\beta=0$ and $\beta=0.5$ for several values of the scaled event horizon velocity $\Omega_{\mathrm{H}} \sqrt{\alpha}$. Both $\Omega_{c}$ and $J$ are given in units set by the mass.

the Kerr frequencies. Here rather large deviations can occur, amounting up to $60 \%$ close to $J / M^{2}=1$ [18]. For $\beta=0$ on the other hand the deviations are smaller and exceed the Kerr values.

\section{CONCLUSIONS}

The main purpose of this work was to provide a more detailed description of the construction and of the (basic) physical properties of the spinning EGBd BHs reported in [18]. These configurations can be viewed as the counterparts of Kerr solutions in the presence of a dilatonic GB term in the gravity action. Our results here, together with those in $[18,20]$ show that such BHs share the basic properties of the GR BHs. However, new features occur as well. The most interesting results of this work are perhaps the ones exhibited in Figs. 4 and 5, which exhibit the domain of existence of the EGBd solutions, both in terms of the GB coupling constant and in terms of the angular momentum. In particular, we find that similar to the static case, the spinning EGBd BHs possess a minimal value of the mass. Moreover, their angular momentum can slightly exceed the Kerr bound.

This work can be continued in many possible directions. Perhaps the most important one is to clarify the issue of stability. For spherically symmetric solutions, (mode) stability was established in [59] for radial perturbations and in [14] for axial perturbations. However, due to the complicated form of the field equations, extending this study to the spinning case will be a highly nontrivial task. In this context, let us remark that, as discussed above, these spinning BHs have an ergoregion. Thus, similar to the Kerr case, they should be afflicted by superradiant instabilities in the presence of (massive) bosonic fields.

Another interesting direction would be to further explore astrophysical signatures of these EGBd BHs. An obvious task here will be to study the geodesics in a systematic way and to compute the shadows of EGBd $\mathrm{BHs}$, contrasting the results with those for the Kerr solution [60].

On the more theoretical side, it would be interesting to extend the solutions in this work to other values of the coupling constant $\gamma$, which enters the exponent in (1), and to look for generic properties. Moreover, one may also search for new BH solutions in more general theories containing (1) as the basic piece. The most obvious example here would be to consider generalizations of the Kerr-Newman BHs in EGBd-Maxwell theory. Also, it would be interesting to consider rotating solutions with different asymptotics, e.g. (anti)-de Sitter asymptotics, by supplementing (1) with a cosmological term.

\section{ACKNOWLEDGMENTS}

We acknowledge support by the DFG Research Training Group 1620 "Models of Gravity". B. K. and J. K. gratefully acknowledge support from FP7, Marie Curie Actions, People, International Research Staff Exchange Scheme (IRSES-606096). E. R. gratefully acknowledges support from the FCT-IF programme via the CIDMA Project No. UID/MAT/04106/2013.

\section{APPENDIX A: THE CRITICAL SOLUTIONS}

As noted in Sec. III A, the spinning solutions BH on the fundamental branch cease to exist for $r_{\mathrm{H}}$ smaller than some critical value $r_{\mathrm{H}}^{(\mathrm{cr})}\left(\alpha, \Omega_{\mathrm{H}}\right)$. 


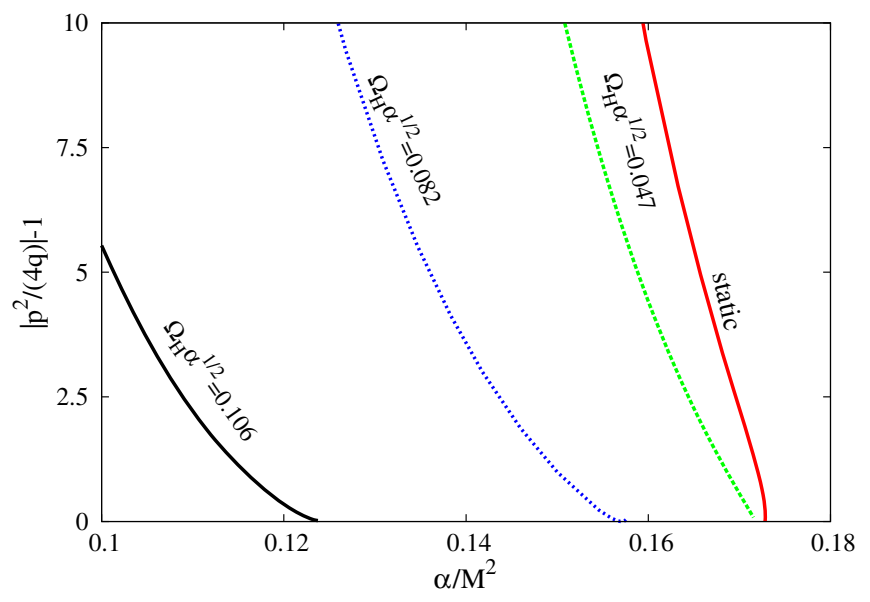

FIG. 14. The quantity $\left|p^{2} /(4 q)\right|-1$ which enters the discriminant $\Delta$ of the equation (A1) is shown as a function of $\alpha / M^{2}$ for several values of the scaled angular velocity $\Omega_{\mathrm{H}} \sqrt{\alpha}$.

To understand this behavior, one has to return to the horizon expansion of the metric functions (17), and look for the expressions of higher order terms. Then, after some algebra, one finds that the second order term $\phi_{2}(\theta)$ in the expansion of the scalar field $\phi(r, \theta)=$ $\phi_{0}(\theta)+\phi_{2}(\theta)\left(r / r_{\mathrm{H}}-1\right)^{2}+\cdots$ is, similar to the static limit, a solution of a quadratic equation,

$$
\phi_{2}^{2}+p \phi_{2}+q=0
$$

where the coefficients $p$ and $q$ depend on $f_{2}(\theta), l_{2}(\theta)$, and $m_{2}(\theta)$, and on their first and second derivatives [62]. Then a real solution to the above equation exists only if $\Delta=p^{2}-4 q>0$. In practice, we have monitored this discriminant for a set of values of $\left(\alpha, \Omega_{\mathrm{H}}\right)$ and observed that the solutions always cease to exist exactly when $\Delta$ becomes negative. This is illustrated in Fig. 14 [63].

We mention that the analogous behavior has been noticed for other nonspherically symmetric solutions with a GB term in the action (see $[64,65]$ ).

\section{APPENDIX B: THE ISSUE OF EXTREMAL SOLUTIONS}

As discussed in Sec. III, the numerical solutions with very small values of the Hawking temperature have smooth metric functions which tend to well-defined limits as $T_{\mathrm{H}} \rightarrow 0$; however, their scalar field takes large values at the poles of the $\mathrm{BH}$ horizon. Thus, by extrapolating this result, it is natural to conjecture that the extremal solution is singular, with a divergent scalar field.

A partial confirmation of this conjecture, together with some analytical understanding, can be achieved when instead of solving the full bulk EGBd equations searching for extremal solutions, one attempts to construct the corresponding near-horizon configurations. There one deals with a codimension one problem, whose solutions are easier to study.

In this approach, following, e.g., [66], one considers the following line element with an isometry group $S O(2,1) \times U(1)$

$$
\begin{aligned}
d s^{2}= & v_{1}(\theta)^{2}\left(-r^{2} d t^{2}+\frac{d r^{2}}{r^{2}}+\beta^{2} d \theta^{2}\right) \\
& +\beta^{2} v_{2}^{2}(\theta)(d \varphi-K r d t)^{2},
\end{aligned}
$$

where $\beta, K$ are real parameters, $0 \leq r<\infty$, while $\theta, \varphi$ and $t$ have the usual range. The above line element would describe the neighborhood of the event horizon of an extremal EGBd BH [67] (and will be an attractor for the full bulk solutions).

The equations satisfied by $v_{i}$ can be derived from the reduced Lagrangian

$$
\begin{aligned}
L= & -2 \beta^{2} v_{1} v_{2}+\beta^{4} \frac{K^{2} v_{2}^{3}}{2 v_{1}}+\frac{2 v_{2} v_{1}{ }^{2}}{v_{1}}+4 v_{1}^{\prime} v_{2}^{\prime} \\
& -\frac{1}{2} v_{1} v_{2} \phi^{\prime 2}+2 \alpha \gamma e^{-\gamma \phi} \frac{\phi^{\prime}}{v_{1}} \\
& \times\left(2 \beta^{2} K^{2} \frac{v_{2}^{3} v_{1}^{\prime}}{v_{1}^{3}}+4 v_{2}^{\prime}-3 \beta^{2} K^{2} \frac{v_{2}^{2} v_{2}^{\prime}}{v_{1}^{2}}+\frac{4 v_{1}^{\prime 2} v_{2}}{\beta^{2} v_{1}^{2}}\right),
\end{aligned}
$$

where a prime denotes a derivative with respect to the angular variable $\theta$. Without any loss of generality, we set $K=1$ in the above relations [69].

The Einstein gravity solution is found for $\alpha=0$, $\phi=$ const. and reads [70]

$$
\begin{aligned}
\beta & =1, \quad v_{1}^{2}=\frac{J}{32 \pi}(3+2 \cos 2 \theta), \\
v_{2}^{2} & =\frac{J}{4 \pi} \frac{\sin ^{2} \theta}{\left(1+\cos ^{2} \theta\right)},
\end{aligned}
$$

with $J$ an arbitrary parameter.

Although we have failed to find an exact solution in the general EGBd case, a solution can be constructed perturbatively in $\alpha$ around the configuration (B3), by taking

$$
\begin{aligned}
\beta & =1+\alpha \beta_{1}+\cdots, \\
v_{1}(\theta) & =v_{10}(\theta)+\alpha v_{11}(\theta)+\cdots, \\
v_{2}(\theta) & =v_{20}(\theta)+\alpha v_{21}(\theta)+\cdots, \\
\phi(\theta) & =\phi_{0}+\alpha \phi_{1}(\theta)+\cdots,
\end{aligned}
$$

where $v_{10}(\theta)$ and $v_{20}(\theta)$ are the Einstein gravity functions. By solving a linear ordinary differential equation, one finds the following expression for $\phi_{1}(\theta)$ : 


$$
\begin{aligned}
\phi_{1}(\theta)= & c_{1} \log \left(\tan \frac{\theta}{2}\right)+c_{2} \\
& +64 e^{-\gamma \phi_{0}} \gamma \pi \frac{(11+20 \cos 2 \theta+\cos 4 \theta)}{J(3+\cos 2 \theta)^{2}} \\
& -32 e^{-\gamma \phi_{0}} \frac{\gamma \pi}{J} \log (3+\cos 2 \theta) \\
& +64 e^{-\gamma \phi_{0}} \frac{\gamma \pi}{J} \log (\sin \theta),
\end{aligned}
$$

with $c_{1}, c_{2}$ constants of integration. One can easily see that, for any choice of the constants $c_{1}, c_{2}$ the function $\phi_{1}(\theta)$ necessarily diverges at $\theta=0$ and/ or $\theta=\pi$.

One may argue that this is an artifact of the first order perturbation theory. However, we have failed to find nonperturbative (numerical) solutions with the required asymptotics. Let us briefly describe our approach. The approximate form of the (putative) solutions as $\theta \rightarrow 0$ is

$$
\begin{aligned}
& v_{1}(\theta)=v_{10}-\frac{1}{4} \beta^{2} v_{10} \theta^{2}+\cdots, \\
& v_{2}(\theta)=v_{10} \theta+\frac{1}{12} \beta^{2} v_{10} \theta^{3}+\cdots, \\
& \phi(\theta)=\phi_{0}+\frac{9 \alpha \gamma \beta^{4} e^{\gamma \phi_{0}} v_{10}^{2}}{192 \alpha^{2} \gamma^{2}-4 e^{2 \gamma \phi_{0}} v_{10}^{4}} \theta^{4}+\cdots
\end{aligned}
$$

A similar expression holds as $\theta \rightarrow \pi$ (here we do not suppose the existence of a reflection symmetry in the equatorial plane), with

$$
\begin{aligned}
& v_{1}(\theta)=\bar{v}_{10}-\frac{1}{4} \beta^{2} \bar{v}_{10}(\pi-\theta)^{2}+\cdots, \\
& v_{2}(\theta)=\bar{v}_{10}(\pi-\theta)+\frac{1}{12} \beta^{2} \bar{v}_{10}(\pi-\theta)^{3}+\cdots, \\
& \phi(\theta)=\bar{\phi}_{0}+\frac{9 \alpha \gamma \beta^{4} e^{\gamma \bar{\phi}_{0}} \bar{v}_{10}^{2}}{192 \alpha^{2} \gamma^{2}-4 e^{2 \gamma \bar{\phi}_{0}} \bar{v}_{10}^{4}}(\pi-\theta)^{4},
\end{aligned}
$$

where $v_{10}$ and $\bar{v}_{10}$ are constants. In this approach, the shooting parameter is $\beta$ and the solutions should exist for a finite range of $v_{10}$ and/or $\bar{v}_{10}$.

In our attempt, one starts with the asymptotics (B6) for some fixed $v_{10}$ and tries to adjust the parameter $\beta$ such that (B7) is smoothly approached as $\theta \rightarrow \pi$. However, for all configurations we have found so far, this was not the case. The scalar field $\phi(\theta)$ is a monotonic function, having a tendency to diverge as $\theta \rightarrow \pi$. This can be understood from the small $\alpha$ expansion (B5), which thus remains valid also within a nonperturbative approach.

The natural interpretation of these results is the absence of the EGBd solutions with a line element of the form (B1) and a regular scalar field. Thus the inclusion of a GBd term in the action does not allow for the rotating BHs to approach extremality and remain regular.
[1] F. Moura and R. Schiappa, Higher-derivative corrected black holes: Perturbative stability and absorption crosssection in heterotic string theory, Classical Quantum Gravity 24, 361 (2007).

[2] T. Kobayashi, M. Yamaguchi, and J. Yokoyama, Generalized G-inflation: Inflation with the most general secondorder field equations, Prog. Theor. Phys. 126, 511 (2011).

[3] E. Berti et al., Testing general relativity with present and future astrophysical observations, Classical Quantum Gravity 32, 243001 (2015).

[4] Interestingly, as shown in [5,6], the EGBd model allows for traversable wormhole solutions, without needing any form of exotic matter.

[5] P. Kanti, B. Kleihaus, and J. Kunz, Wormholes in Dilatonic Einstein-Gauss-Bonnet Theory, Phys. Rev. Lett. 107, 271101 (2011).

[6] P. Kanti, B. Kleihaus, and J. Kunz, Stable Lorentzian wormholes in dilatonic Einstein-Gauss-Bonnet theory, Phys. Rev. D 85, 044007 (2012).

[7] In contrast to other models possessing solutions with scalar hair, the (pure) Einstein gravity BHs do not satisfy the field equations of the full (EGBd) model.

[8] S. Mignemi and N. R. Stewart, Charged black holes in effective string theory, Phys. Rev. D 47, 5259 (1993).
[9] S. Mignemi, Dyonic black holes in effective string theory, Phys. Rev. D 51, 934 (1995).

[10] P. Kanti, N. E. Mavromatos, J. Rizos, K. Tamvakis, and E. Winstanley, Dilatonic black holes in higher curvature string gravity, Phys. Rev. D 54, 5049 (1996).

[11] T. Torii, H. Yajima, and K. i. Maeda, Dilatonic black holes with Gauss-Bonnet term, Phys. Rev. D 55, 739 (1997).

[12] S. O. Alexeyev and M. V. Pomazanov, Black hole solutions with dilatonic hair in higher curvature gravity, Phys. Rev. D 55, 2110 (1997).

[13] Z. K. Guo, N. Ohta, and T. Torii, Black holes in the dilatonic Einstein-Gauss-Bonnet theory in various dimensions. I. Asymptotically flat black holes, Prog. Theor. Phys. 120, 581 (2008).

[14] P. Pani and V. Cardoso, The case for Einstein-DilatonGauss-Bonnet black holes, Phys. Rev. D 79, 084031 (2009).

[15] A. Maselli, P. Pani, L. Gualtieri, and V. Ferrari, Rotating black holes in Einstein-Dilaton-Gauss-Bonnet gravity with finite coupling, Phys. Rev. D 92, 083014 (2015).

[16] P. Pani, C. F. B. Macedo, L. C. B. Crispino, and V. Cardoso, Slowly rotating black holes in alternative theories of gravity, Phys. Rev. D 84, 087501 (2011).

[17] D. Ayzenberg and N. Yunes, Slowly-rotating black holes in Einstein-Dilaton-Gauss-Bonnet gravity: Quadratic order in 
spin solutions, Phys. Rev. D 90, 044066 (2014); 91, 069905 (2015).

[18] B. Kleihaus, J. Kunz, and E. Radu, Rotating Black Holes in Dilatonic Einstein-Gauss-Bonnet Theory, Phys. Rev. Lett. 106, 151104 (2011).

[19] The specific angular momentum $a$ of Kerr BHs is bounded by $|a| \leq M$ with $a=J / M$.

[20] B. Kleihaus, J. Kunz, and S. Mojica, Quadrupole moments of rapidly rotating compact objects in dilatonic EinsteinGauss-Bonnet theory, Phys. Rev. D 90, 061501(R) (2014).

[21] In this work we shall use geometric units $c=G=1$.

[22] This term alone does not yield modifications of Einstein's equations, its integral leading to a boundary term in the action. This is no longer the case, however, once $R_{\mathrm{GB}}^{2}$ couples with dynamical matter fields.

[23] In this work we shall treat $\alpha$ as an arbitrary input constant. However, note that observations of $\mathrm{BH}$ low-mass X-ray binaries give a constraint $\sqrt{\alpha} \lesssim 5 \times 10^{6} \mathrm{~cm}$ [24], which is comparable to the typical size of a stellar-mass $\mathrm{BH}$, see the discussion in [3].

[24] K. Yagi, A new constraint on scalar Gauss-Bonnet gravity and a possible explanation for the excess of the orbital decay rate in a low-mass X-ray binary, Phys. Rev. D 86, 081504 (2012).

[25] R. M. Wald, General Relativity (Chicago University Press, Chicago, 1984)

[26] B. Kleihaus and J. Kunz, Rotating Hairy Black Holes, Phys. Rev. Lett. 86, 3704 (2001); B. Kleihaus, J. Kunz, and F. Navarro-Lerida, Rotating Einstein-Yang-Mills black holes, Phys. Rev. D 66, 104001 (2002).

[27] Setting $\left.\phi\right|_{r=\infty}=0$ removes the scaling symmetry of the equations $\phi \rightarrow \phi+c, r \rightarrow r e^{-\gamma c / 2}$.

[28] Note that, similar to the pure Einstein gravity case, the equation $E_{r}^{\theta}=0$ implies that the ratio $f_{2}^{2} / m_{2}$ is constant, a supplementary condition which is used as another test of the numerical accuracy. This also implies the constancy of the Hawking temperature, as given by (25).

[29] The absence of conical singularities implies as well $\left.m\right|_{\theta=0, \pi}=\left.l\right|_{\theta=0, \pi}$.

[30] R. M. Wald, Black hole entropy is the Noether charge, Phys. Rev. D 48, R3427 (1993).

[31] For example, one uses the observation that both $E_{\varphi}^{t} \sqrt{-g}$ and $\left(R_{t}^{t}+\alpha e^{-\gamma \phi}\left(T_{t}^{t(\mathrm{GBd})}+\frac{1}{2} R_{\mathrm{GB}}^{2}\right)\right) \sqrt{-g}$ are total derivatives (i.e., they have expressions of the form $\partial_{r} U_{r}+\partial_{\theta} U_{\theta}$ with $U_{r}, U_{\theta}$ complicated functions of $\mathcal{F}_{i}$ and their derivatives). Moreover, $e^{-\gamma \phi} T_{\mu}^{\mu(\mathrm{GBd})} \sqrt{-g}$ can also be written as a total derivative.

[32] Note that, however, due to the contribution of the GaussBonnet term, the final equations are not diagonal with respect to second derivatives of $\mathcal{F}_{i}$.

[33] W. Schönauer and R. Weiß, Efficient vectorizable PDE solvers, J. Comput. Appl. Math. 27, 279 (1989); M. Schauder, R. Weiß, and W. Schönauer, Universität Karlsruhe, Interner Bericht Nr. Report No. 46/92, 1992.

[34] C. A. R. Herdeiro and E. Radu, Asymptotically flat black holes with scalar hair: A review, Int. J. Mod. Phys. D 24, 1542014 (2015).

[35] Note, that for $\gamma=1$ the minuscule second branch exists only for $0.17284 \leq \frac{\alpha}{M^{2}} \lesssim 0.17285$ and can thus be neglected unless high accuracy is demanded.
[36] In principle, it is sufficient to consider a single value of $\Omega_{\mathrm{H}}$ and scan the full range of $\alpha$. In practice, however, some regions of the parameter space are explored more easily by varying both $\Omega_{\mathrm{H}}$ and $\alpha$.

[37] However, for sufficiently large values of $\Omega_{\mathrm{H}} \sqrt{\alpha}$ (e.g., $\Omega_{\mathrm{H}} \sqrt{\alpha}=0.11$ ) one finds a single branch of solutions, with $0<r_{\mathrm{H}}<r_{\mathrm{H}}^{(\mathrm{cr})}$.

[38] Note, that only near extremal solutions can be constructed within the framework proposed in this work.

[39] Much larger violations of the Kerr bound have been found recently in a model containing a massive complex scalar field minimally coupled to Einstein gravity [40,41,46] (see also the more general discussion in [42]). There, the violation can be related to the existence of a solitonic limit of the BHs, which is not the case for the EGBd model. We conjecture that the violation of the Kerr bound for the solutions in this work can be attributed to the existence of regions with a negative effective energy density, as obtained from (6). (A discussion of this feature is given in [10] for the spherically symmetric limit.).

[40] C. A. R. Herdeiro and E. Radu, Kerr Black Holes with Scalar Hair, Phys. Rev. Lett. 112, 221101 (2014).

[41] C. Herdeiro and E. Radu, Construction and physical properties of Kerr black holes with scalar hair, Classical Quantum Gravity 32, 144001 (2015).

[42] C. A. R. Herdeiro and E. Radu, How fast can a black hole rotate?, Int. J. Mod. Phys. D 24, 1544022 (2015).

[43] This can be seen, e.g., from the expression (22). Note that the functions $m_{2}(\theta), l_{2}(\theta)$, and $f_{2}(\theta)$ are strictly positive. However, one should remark that it is not a priori clear that the all BHs in EGBd theory would necessarily possess a horizon with $S^{2}$ topology, since a number of classical GR theorems do not apply to this model.

[44] L. Smarr, Surface geometry of charged rotating black holes, Phys. Rev. D 7, 289 (1973).

[45] It is interesting to note that this behavior strongly contrasts with the results found for the spinning BHs with scalar hair in $[40,41,46]$. There, the scalar field is complex and carries a Noether charge, whose density is proportional to the angular momentum density. Also, for those solutions $T_{t}^{t(\phi)}$ does not vanish in the equatorial plane.

[46] B. Kleihaus, J. Kunz, and S. Yazadjiev, Scalarized hairy black holes, Phys. Lett. B 744, 406 (2015).

[47] C. Herdeiro and E. Radu, Ergosurfaces for Kerr black holes with scalar hair, Phys. Rev. D 89, 124018 (2014).

[48] This is not a real surprise, since the presence of an ergo-torus for the solutions in $[40,46]$, can be traced back to the existence of a (spinning) solitonic limit with an ergo-torus [49], which is not the case for the EGBd theory.

[49] B. Kleihaus, J. Kunz, M. List, and I. Schaffer, Rotating boson stars and Q-balls. II. Negative parity and ergoregions, Phys. Rev. D 77, 064025 (2008).

[50] A. E. Broderick, T. Johannsen, A. Loeb, and D. Psaltis, Testing the no-hair theorem with event horizon telescope observations of Sagittarius A*, Astrophys. J. 784, 7 (2014).

[51] The quadrupole moment is extracted following Geroch and Hansen [52,53] (see also [54,55]).

[52] R. P. Geroch, Multipole moments. II. Curved space, J. Math. Phys. (N.Y.) 11, 2580 (1970). 
[53] R. O. Hansen, Multipole moments of stationary space-times, J. Math. Phys. (N.Y.) 15, 46 (1974).

[54] C. Hoenselaers and Z. Perjes, Multipole moments of axisymmetric electrovacuum spacetimes, Classical Quantum Gravity 7, 1819 (1990).

[55] T. P. Sotiriou and T. A. Apostolatos, Corrected multipole moments of axisymmetric electrovacuum spacetimes, Classical Quantum Gravity 21, 5727 (2004).

[56] In the Kerr case the coefficients are $M_{2}=-2 M\left(M^{2}-4 r_{\mathrm{H}}^{2}\right) / 3$ and $C_{1}=-2 r_{\mathrm{H}}^{2}$.

[57] B. Carter, Global structure of the Kerr family of gravitational fields, Phys. Rev. 174, 1559 (1968).

[58] This agrees with the perturbative results in [17].

[59] P. Kanti, N. E. Mavromatos, J. Rizos, K. Tamvakis, and E. Winstanley, Dilatonic black holes in higher curvature string gravity. 2: Linear stability, Phys. Rev. D 57, 6255 (1998).

[60] For example, the results in the recent work [61] show that the shadow of a spinning BH with scalar hair (for a model with a massive complex scalar field) can be drastically different as compared to the GR results.

[61] P. V. P. Cunha, C. A. R. Herdeiro, E. Radu, and H. F. Runarsson, Shadows of Kerr Black Holes with Scalar Hair, Phys. Rev. Lett. 115, 211102 (2015).

[62] We recall that the ratio $f_{2}^{2} / m_{2}$ is constant [see relation (25)].

[63] We note, that for the spinning solutions we did not observe a minuscule branch whose mass increases when the horizon size decreases, as present in the static case. While by continuity, such a branch should be present for sufficiently slowly rotating $\mathrm{BHs}$, its uncovering would need rather high numerical accuracy.

[64] B. Kleihaus, J. Kunz, and E. Radu, Generalized Weyl solutions in $d=5$ Einstein-Gauss-Bonnet theory: The static black ring, J. High Energy Phys. 02 (2010) 092.

[65] B. Kleihaus, J. Kunz, E. Radu, and B. Subagyo, Spinning black strings in five dimensional Einstein-Gauss-Bonnet gravity, Phys. Lett. B 713, 110 (2012).

[66] D. Astefanesei, K. Goldstein, R. P. Jena, A. Sen, and S. P. Trivedi, Rotating attractors, J. High Energy Phys. 10 (2006) 058 .

[67] It is interesting to note that, as discussed in [68] in a different context, the existence of the near horizon solution does not guarantee the existence of a corresponding bulk configuration.

[68] J. L. Blazquez-Salcedo, J. Kunz, F. Navarro-Lerida, and E. Radu, Sequences of extremal radially excited rotating black holes, Phys. Rev. Lett. 112, 011101 (2014); J. L. BlazquezSalcedo, J. Kunz, F. Navarro-Lerida, and E. Radu, Radially excited rotating black holes in Einstein-Maxwell-ChernSimons theory, Phys. Rev. D 92, 044025 (2015).

[69] This is a result of the scaling symmetry $v_{2} \rightarrow \lambda v_{2}$, $K \rightarrow K / \lambda$.

[70] J. M. Bardeen and G. T. Horowitz, The extreme Kerr throat geometry: A vacuum analog of $\operatorname{Ads}(2) \times S^{2}$, Phys. Rev. D 60, 104030 (1999). 